\title{
Challenges for and Pathways toward Solid-State Batteries
}

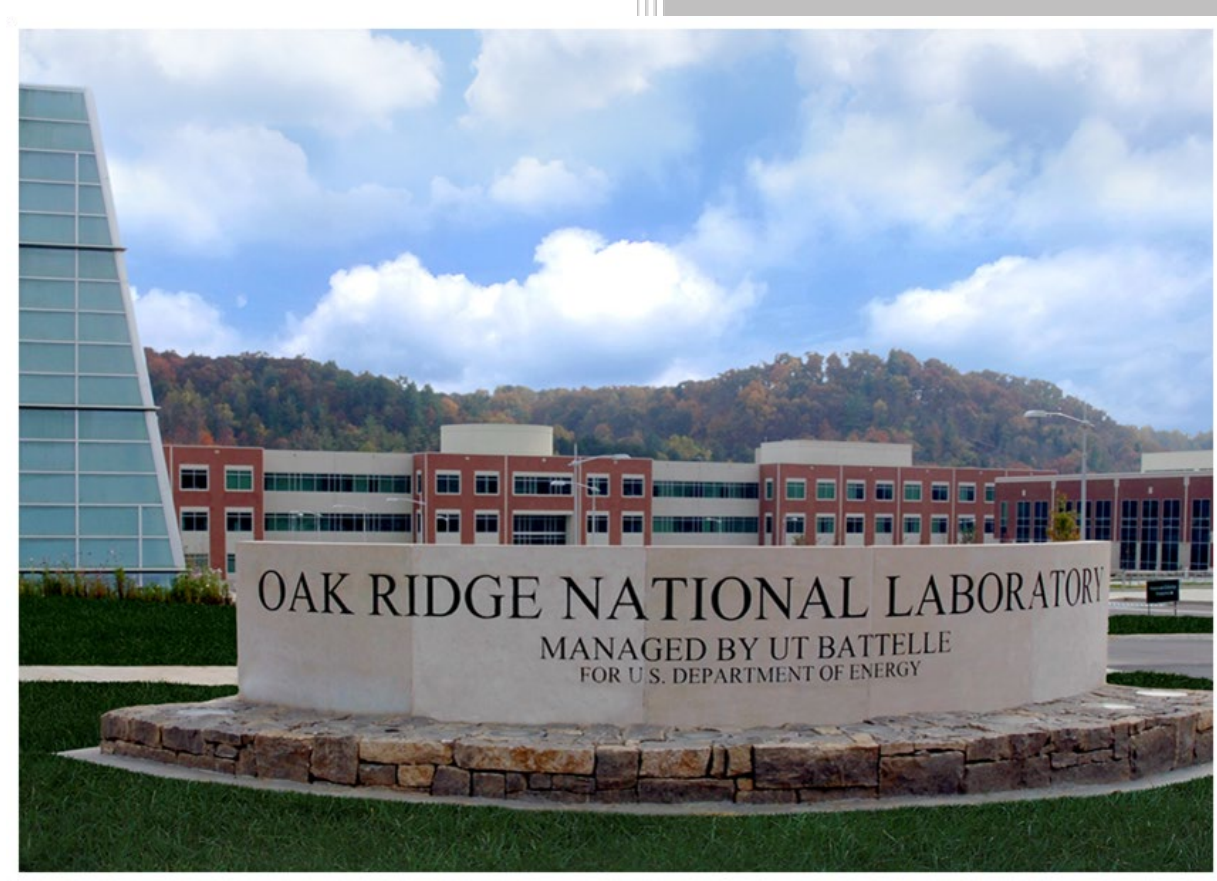

Approved. For public release

September 30, 2020 


\section{DOCUMENT AVAILABILITY}

Reports produced after January 1, 1996, are generally available free via US Department of Energy (DOE) SciTech Connect.

Website www.osti.gov

Reports produced before January 1, 1996, may be purchased by members of the public from the following source:

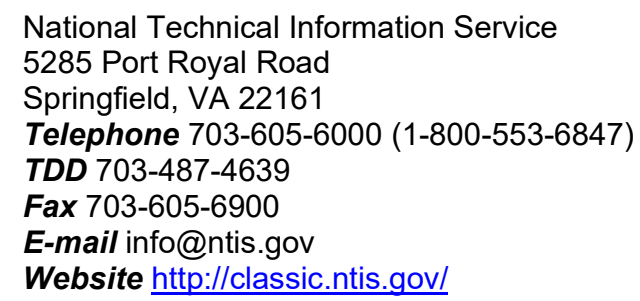

Reports are available to DOE employees, DOE contractors, Energy Technology Data Exchange representatives, and International Nuclear Information System representatives from the following source:

Office of Scientific and Technical Information

PO Box 62

Oak Ridge, TN 37831

Telephone 865-576-8401

Fax 865-576-5728

E-mail reports@osti.gov

Website http://www.osti.gov/contact.html

This report was prepared as an account of work sponsored by an agency of the United States Government. Neither the United States Government nor any agency thereof, nor any of their employees, makes any warranty, express or implied, or assumes any legal liability or responsibility for the accuracy, completeness, or usefulness of any information, apparatus, product, or process disclosed, or represents that its use would not infringe privately owned rights. Reference herein to any specific commercial product, process, or service by trade name, trademark, manufacturer, or otherwise, does not necessarily constitute or imply its endorsement, recommendation, or favoring by the United States Government or any agency thereof. The views and opinions of authors expressed herein do not necessarily state or reflect those of the United States Government or any agency thereof. 
Chemical Sciences Division and

Energy and Transportation Science Division

\section{CHALLENGES FOR AND PATHWAYS TOWARD SOLID-STATE BATTERIES}

Date Published: November 17, 2020

Prepared by OAK RIDGE NATIONAL LABORATORY

Oak Ridge, TN 37831-6283 managed by UT-BATTELLE, LLC for the US DEPARTMENT OF ENERGY under contract DE-AC05-00OR22725 



\section{CONTENTS}

CONTRIBUTORS

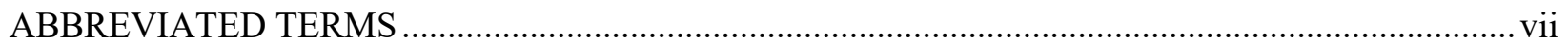

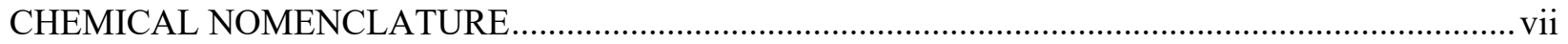

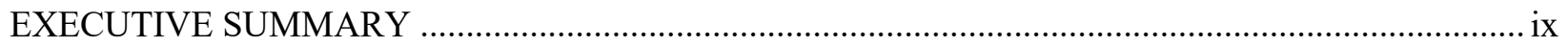

1. CHALLENGES FOR AND PATHWAYS TOWARD SOLID-STATE BATTERIES ...................... 1

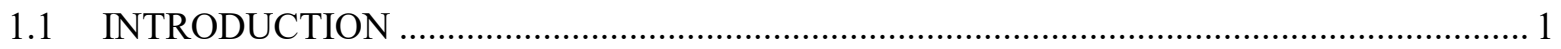

1.2 THE SCIENCE GAPS IN STUDY OF MATERIALS AND INTERFACES .......................... 4

1.2.1 Science Gaps for the Solid Electrolyte in Contact with Li Metal ................................... 6

1.2.2 Science Gaps for Active Cathode Materials and Solid-State Composite

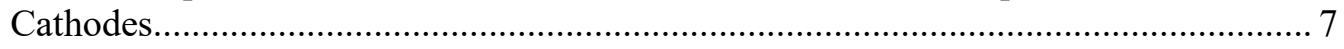

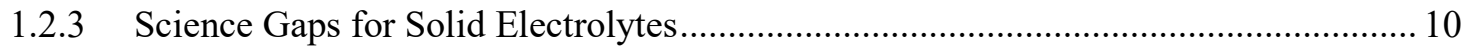

1.3 THE KNOWLEDGE GAPS IN THE SCIENCE OF PROCESSING …................................. 11

1.4 KNOWLEDGE GAPS AND OPPORTUNITIES TO DESIGN BATTERY

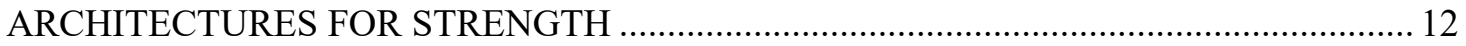

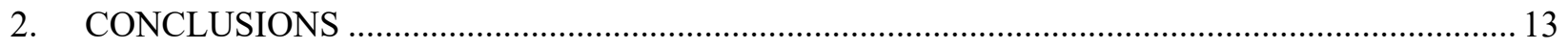

2.1 PATHWAYS TO SOLID-STATE BATTERIES - ADDRESSING SCIENCE GAPS ........... 13

2.1.1 Study Control and Efficient Cycling of the Li Metal Anode .................................... 13

2.1.2 Study to Advance Dry, Solid-State Cathodes and Composites.................................... 14

2.1.3 Solid-State Battery Development with Rapid Integration of Electrode and

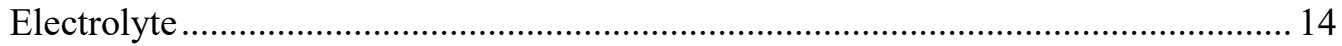

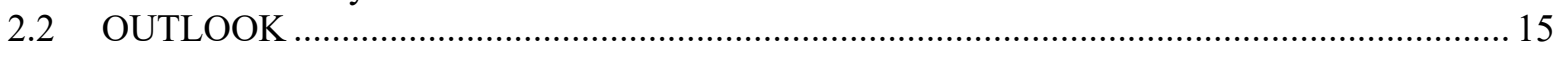

APPENDIX A. WORKSHOP PROGRAM ….................................................................................. A-1

APPENDIX B. TWO SLIDES SUBMITTED BY EACH PARTICIPANT BEFORE WORKSHOP ..... B-1

APPENDIX C. KEY TOPICS FROM SSB WORKSHOP ….......................................................... 



\section{CONTRIBUTORS}

\section{Organizations}

Applied Materials
Argonne National Laboratory
Ford Motor Company
General Motors
Georgia Institute of Technology
Iowa State University
Lawrence Berkeley National Laboratory
Michigan Technological University
Oak Ridge National Laboratory

Pacific Northwest National Laboratory Solid Power

University of Colorado at Boulder University of California at Berkeley University of California at Los Angeles University of California at San Diego University of Maryland

University of Michigan

\section{Contributors}

Subra Herle

Zonghei Chen, Joseph Libera, Sanja Tepavcevic

Venkat Anandan

Thomas Yersak

Matthew McDowell

Steve Martin

Marca Doeff, Steven Harris

Erik Herbert

Ilias Belharouak, Claus Daniel, Nancy Dudney, Sergiy Kalnaus, Jagjit Nanda, Ethan Self, Andrew Westover

Dongping Lu, Jie Xiao

Josh Buettner-Garrett

Chunmei Ban

Nitash Balsara, Bryan McCloskey

Bruce Dunn

Shirley Meng

Paul Albertus, Eric Wachsman, Chunsheng Wang

Jeff Sakamoto 



\section{ABBREVIATED TERMS}

ARPA-E Advanced Research Projects Agency_Energy

Battery500 Consortium led by Pacific Northwest National Laboratory and sponsored by DOE VTO

DOE US Department of Energy

EV electric vehicle

KPFM Kelvin probe force microscopy

ORNL Oak Ridge National Laboratory

PI principal investigator

SE $\quad$ solid electrolyte

SECCM scanning electrochemical cell microscopy

SSB solid-state battery

\section{CHEMICAL NOMENCLATURE}

Lipon amorphous lithium phosphorous oxynitride, typically near $\mathrm{Li}_{3.4} \mathrm{PO}_{3} \mathrm{~N}_{0.6}$

LLTO $\quad \mathrm{Li}_{3 x} \mathrm{La}_{2 / 3-x} \mathrm{TiO}_{3}$

LLZO lithium lanthanum zirconium oxide, nominally $\mathrm{Li}_{7} \mathrm{La}_{3} \mathrm{Zr}_{2} \mathrm{O}_{12}$ with $\mathrm{Al}$, $\mathrm{Ta}$, or other dopants.

LPS $\quad \mathrm{Li}_{3} \mathrm{PS}_{4}$ (doped or undoped). This may also include amorphous or crystalline phases.

MD molecular dynamics

NMC lithium nickel manganese cobalt oxide, a high-voltage intercalation compound of $\mathrm{Li}(\mathrm{Ni}, \mathrm{Mn}, \mathrm{Co}) \mathrm{O}_{2}$, with emphasis on the Ni-rich, low-Co compositions such as an 811 ratio.

LCO $\quad \mathrm{LiCoO}_{2}$

LFP $\quad \mathrm{LiFePO}_{4}$

SEO $\quad\left(\mathrm{SEEO}^{\mathrm{TM}}\right)$ is block co-polymer of polystyrene poly(ethylene oxide), with a dissolved Li salt, such as lithium bis(trifluoromethane)sulfonimide (LiTFSI). 



\section{EXECUTIVE SUMMARY}

Solid-state batteries utilizing lithium metal anodes have the potential to enable batteries with a specific energy of $>500 \mathrm{Wh} / \mathrm{kg}$ and an energy density of $>1,500 \mathrm{Wh} / \mathrm{L}$ for thousands of cycles. When optimized they will improve the energy efficiency, operating temperature range, sustainability, and safety at a lower cost compared to projections for advanced Li-ion batteries. This improved performance is critical for the widespread adoption of electric vehicles and may enable future applications such as electric aviation. Expectations for solid-state batteries are high, there are significant materials and processing challenges that need to be overcome. Some of these challenges are well known; others are more subtle and are just becoming known. The challenges and their solutions must be clearly identified to realize high-energy solid-state Li metal batteries.

In the United States, the U.S. Department of Energy (DOE) funds the majority of energy storage research and development, including solid state battery research. Web of Science publication analysis shows that worldwide DOE is second largest funder. Work on solid state batteries is distributed across different arms of DOE and is coordinated at headquarters to ensure a broad national portfolio for advanced batteries, including lithium batteries and solid-state battery devices. We note that university and national laboratory researchers in the US with a long-standing interest in solid state batteries is a small, integrated community with recognized impact for publications, patents and startup companies. The opportunity for this group to engage for research with international colleagues is growing, fostered by periodic bilateral meetings supported by DOE and by the recent joint US German research program for lithium battery interface studies. These are valuable opportunities to speed solid-state battery development. The ORNL led virtual workshop, overviewed here, confirms the community's shared vision of the exciting advances, opportunities, and challenges. Further, we are seeing that this workshop has spawned several new informal collaborations and draft proposals. This encourages us to propose future discussion.

On May 11, 2020, Oak Ridge National Laboratory (ORNL) hosted a 6-hour, on-line national workshop to discuss recent advances and most the prominent obstacles to realizing solid-state Li metal batteries. The workshop included more than 30+ experts from national laboratories, universities, and companies, all of whom have worked on solid-state batteries for multiple years. The participants shared recent advances, many not yet in print, illustrating that the community has gained significant new insights for solid-state Li battery materials over the last 5 years. Several advances are given in the report, including the following examples.

- Revision of the Monroe Newman model suggests conditions where the Li plating leads to a smoother interface than Li filament growth.

- Local deformation does not initiate fracture in the amorphous Lipon (lithium phosphorous oxynitride) electrolyte as generally occurs for ceramic electrolytes.

- A highly conducting and robust catholyte was formed by filling a cathode array with sol-gel precursors followed by thermal curing.

The consensus of the participants is that, although the progress is exciting, much has yet to be researched and discovered. Our goal was to examine the issues and identify the most pressing needs and biggest opportunities. Workshop participants were asked to present their views by articulating fundamental knowledge gaps for materials science, processing science, and battery architectures that are critical to advancing solid-state battery technology. This input was used to set the workshop agenda. Also, the group considered what would incentivize the adoption for US manufacturing and how to accelerate and focus research attention for the greater benefit of the US energy, climate, and economic interests. We believe 
that there are advantages for highly integrated collaborative projects in addition to independent smallgroup projects at this stage in solid-state battery activities. Ongoing projects have proven successful, and good paths should not be abandoned; however, it is not too early for a collaboration to seek the ultimate energy density and lifetime that may be possible with solid-state batteries. We identified pros and cons for each of the major types of solid-state cells (i.e., cells based on sulfide, oxide, or polymer solid electrolytes), but we also recognize common science gaps that bridge the different chemistries. Addressing the science gaps identified during the workshop may reveal the most promising systems to pursue in the future.

The workshop participants also recognized that much of the leading scientific and development work taking place on solid-state batteries is occurring in Japan, South Korea, and Germany. Large efforts are also underway in China, both at universities and in companies. Currently the US federal government has no programs focused directly on advancing solid-state battery devices, as the major battery efforts are for advanced Li-ion and Li battery technology using traditional liquid electrolytes, and for novel energy

storage materials. These programs are the Joint Center for Energy Storage Research, through the US Department of Energy (DOE) Office of Basic Energy Sciences, and Battery500 through the DOE Vehicle Technologies Office. The recommendations in this report help inform the key scientific priorities if an effort focused of solid-state devices were initiated within DOE.

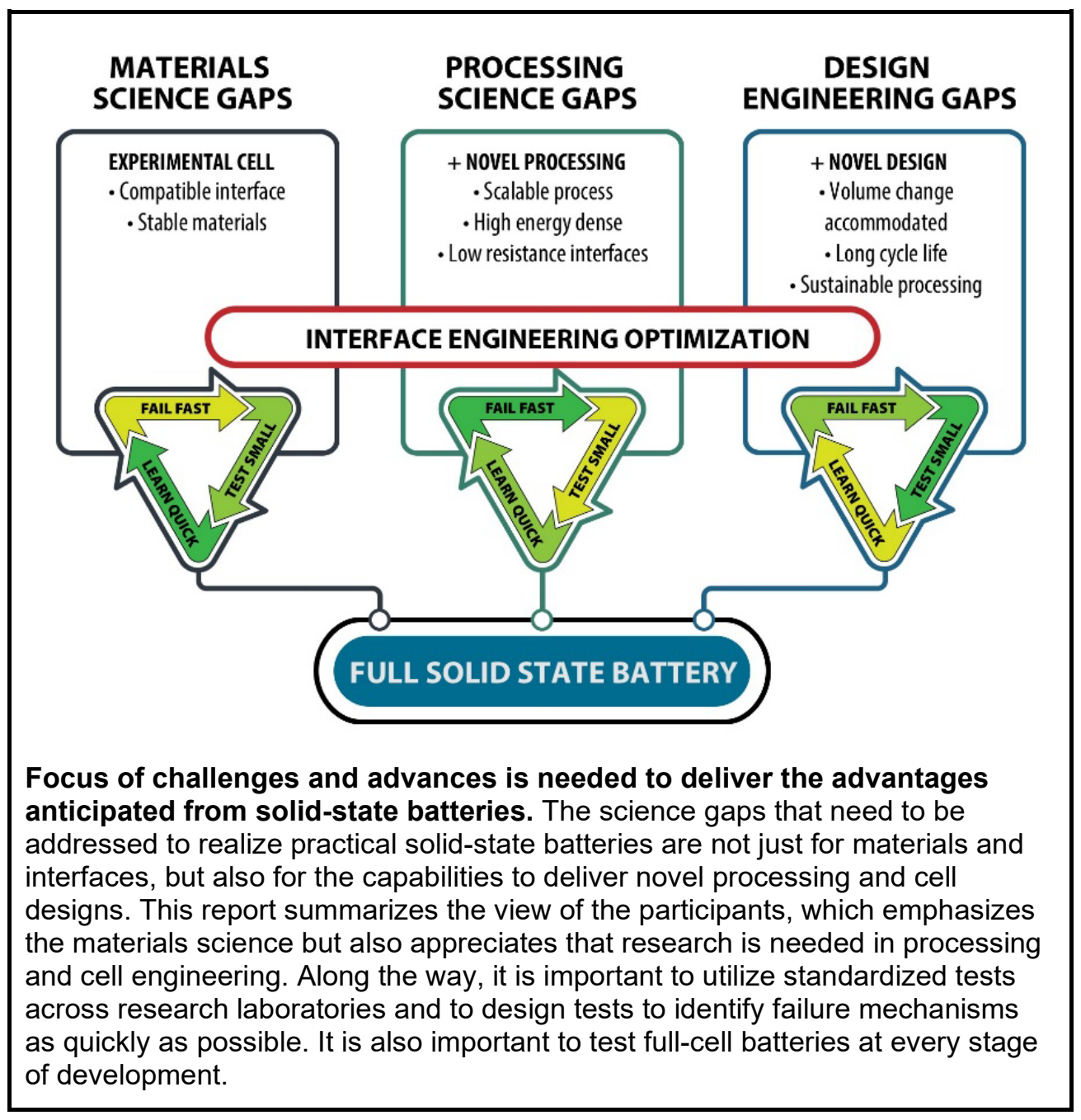


In this report, the major outcomes of the workshop are organized to identify the gaps in our scientific knowledge for four core materials science areas: (1) Li metal anodes, (2) the solid electrolyte in contact with Li metal, (3) active cathode materials and solid-state composite cathodes, and (4) solid electrolytes. Illustrative examples and discussions are reported with the more comprehensive issues in the report. Discussion of additional challenges related to processing of solid-state battery materials and to the designs and architectures for mechanically robust, long-lived batteries received less attention due to time constraints. These are good themes for deeper discussion at a follow-on workshop, where a complete list of critical research topics can be identified.

The following list of science gaps and discussion highlights, selected from the complete list in the report, illustrates the science challenges where deeper understanding of materials and processes may advance the technology.

- Science gaps for Li metal anodes

- What defect generation/annihilation processes operate in $\mathrm{Li}$ films $(<30 \mu \mathrm{m}$ thick $)$ when $\mathrm{Li}$ is plated and stripped through a generic solid electrolyte?

- Is a seed layer of Li the best way to template the plated $\mathrm{Li}$ ?

- Science gaps for the solid electrolyte in contact with Li metal

- What promotes electrochemical stability or efficient passivation with Li?

- How does the Li anode respond to different cathodes across a solid electrolyte separator?

- Science gaps for active cathode materials and solid-state composite cathodes

- What are the relative pressure and electrochemical driving forces experienced by the active cathode material? Is there a restoring component that can provide homogeneous reaction?

- What can be achieved through defect and microstructure engineering to enhance kinetics for a dense, zero-strain cathode membrane?

- Science gaps for solid electrolytes

- How are the battery polarization and resistance affected by inserting a single-ion electrolyte barrier layer at cathodes containing a binary electrolyte?

- What materials-strengthening mechanisms can be adopted for micron-scale thin electrolyte membranes?

- Discussion highlights of science gaps for processing materials and interfaces

- What novel, solvent-free processing routes can be envisioned to form intimate interface contacts?

- What energy sources effectively couple to wide bandgap electrolyte materials for rapid annealing or sintering?

- Discussion highlights for knowledge gaps in battery design and architecture

- Are there design strategies to ensure that the interfaces remain under compression during battery cycling involving differing spatiotemporal volume changes across the cell?

Discussions during and following the workshop led the participants to suggest areas where coordinated US DOE research programs, including multiple principal investigators and institutions, may accelerate progress in research and development of materials for solid-state batteries, particularly for electric vehicles. Two topics address individual components and interfaces for a solid-state battery; the third is a path to integrating all the components for a full cell.

\section{Study Control and Efficient Cycling of the Li Metal Anode}

The driver for solid-state batteries is the attainment of high energy density based on efficient cycling and confinement of the metallic $\mathrm{Li}$ anode. That outcome could be impossible if $\mathrm{Li}$ cannot be confined without application of high stack pressure $(>\sim 1 \mathrm{MPa})$. Although the reasons for poor Li cycling with solid electrolytes are becoming clearer, the solutions are not. It would advance our understanding to clarify the intrinsic/extrinsic properties of the Li metal; the solid electrolyte; and the equilibrated, fully contacted 
interface. In addition to the recognized failure modes, namely growth of Li filaments and voids at the interface, key issues for deeper study include interface passivation in a full cell versus a $\mathrm{Li} /$ solid electrolyte/Li cell, effects of impurities in the $\mathrm{Li}$, properties of the $\mathrm{Li}$ from various processing methods (e.g., rolled, vapor-deposited, electrodeposited), and redistribution of Li with extended cycling at all length scales. To quickly assess the failure and degradation mechanisms of Li anodes, it is important to embrace methods for which the excess Li inventory and the excess volume of the solid electrolyte are nil.

\section{Study to Advance Dry, Solid-State Cathodes and Composites}

Study of the cathode component of a solid-state battery is critical and foundational in several ways. For a battery with the highest energy density, the active cathode should occupy by far the largest fraction of the battery, and as such, the composite cathode should act as the physical support for the battery. The cathode study is also a foundational research topic because it requires solutions for many of the critical interface science challenges. Within the cathode, the effects of volume changes, interface integrity, and phase connectivity for facile ion and electron transport need to be addressed. In a collaborative program, experts in battery materials and solid-state electrochemistry will work closely with experts in materials mechanics to minimize and relieve the stresses from cycling, identify mechanisms and architectures for strengthening the materials and interfaces, utilize materials and processing to form rapid and direct transport paths for ions and electrons that span the thickness of the cathode, and reduce or eliminate the need for external stack pressure.

\section{Solid-State Battery Development with Rapid Integration of Electrodes and Electrolyte}

There are several good choices for materials to use as the solid electrolyte, catholyte, and active cathode components. The best choices for a solid-state battery are not obvious, and the design space is large. This results to a great extent from the traditional research direction, where each material is investigated and refined separately before an attempt is made to fabricate a half cell or a full cell. As an alternative, rather than spending time investigating and refining each material or component, researchers could work on a highly collaborative research activity in which half cells are fabricated at the earliest possible time. The components that must work together are then developed together. In that way, the intrinsic stability and processes for integration are the first considerations. For ambitious goals to re-envision a solid-state battery with maximum energy density, this might be an approach to rapidly down-select the most promising set of materials for a solid-state battery. This approach is only efficient where experts in different classes of materials and in different processing methods work together with experts in mechanical engineering, modeling, and design in a holistic approach leading to a rational approach in which criteria for the entire battery are considered. Unless a deliberate effort is undertaken to narrow the choices, a large program may be fractured, much the same as we have now, with multiple promising solid electrolytes and battery chemistries being pursed at once within individual programs.

Other reasons for a large integrated program and keys for its success, as discussed at the workshop, include having experienced technical associates build and test the batteries, accessing many processing and characterization tools along with experienced operators, sharing of materials and standardized methods for rigorous comparisons, and the free sharing of failures and difficulties among the participants in addition to the successes shared publicly as journal publications.

Because the first workshop proved to be efficient with time and expense, we propose convening a second workshop to further inform recommendations that emphasize the mechanical aspects of solid-state batteries along with novel processing methods to create new materials or advanced battery designs. The recommendation for a follow-up workshop is consistent with a literature survey, conducted at ORNL after the workshop, which determined that processing science and solid-state mechanics are underemphasized in publications on the topic of solid-state batteries. 


\section{CHALLENGES FOR AND PATHWAYS TOWARD SOLID-STATE BATTERIES}

\subsection{INTRODUCTION}

This workshop gathered participants from US national laboratories, universities, and industry, all of whom have long-standing activities in solid-state battery research, with interest in batteries that would be practical and advantageous for electric vehicle (EV) applications. With careful design, solid-state batteries have the promise to improve the energy density and efficiency, lifetime, operating temperature range, and cost of rechargeable lithium batteries. In addition, advances in safety and recycling are likely. Achieving these goals requires efficient use of Li metal as the anode and on reducing the amount of inactive materials throughout the battery. The purpose of the workshop was to exchange information and to determine if this community has developed a consensus on the more promising opportunities to advance solid-state batteries and on the key challenges in materials science, processing science, and design engineering where a focused and expanded effort would have the greatest impact.

In preparation for the workshop, each participant submitted two slides, which were shared several days prior to the virtual workshop. The first slide was an introduction to their team with notes on novel capabilities and expertise available, or being developed, for solid-state batteries. In addition, the principal investigator (PI) identified recommended publications, one from the PI's program and one from the general literature, that were particularly impactful to the field. For the second slide, each PI summarized the critical science gaps that are barriers to advancing materials and interfaces, processing, or battery performance. The agenda was organized from thee suggested challenges (see Appendix A). The slides are shown in Appendix B. Each session in the workshop, following the brief self-introductions, started with a keynote overview from a participant, then 6 to 10 relevant contributions from other participants ( 1 slide each), followed by an open discussion. The proceedings were recorded.

Exciting innovations and observations were reported in the introductions and throughout the workshop. The highlights in the following list show that research continues to discover new materials, identify key mechanisms, and reveal surprising properties that advance the field and provide additional alternatives for practical solid-state batteries. This is an exciting and rapidly growing area of intense investigation.

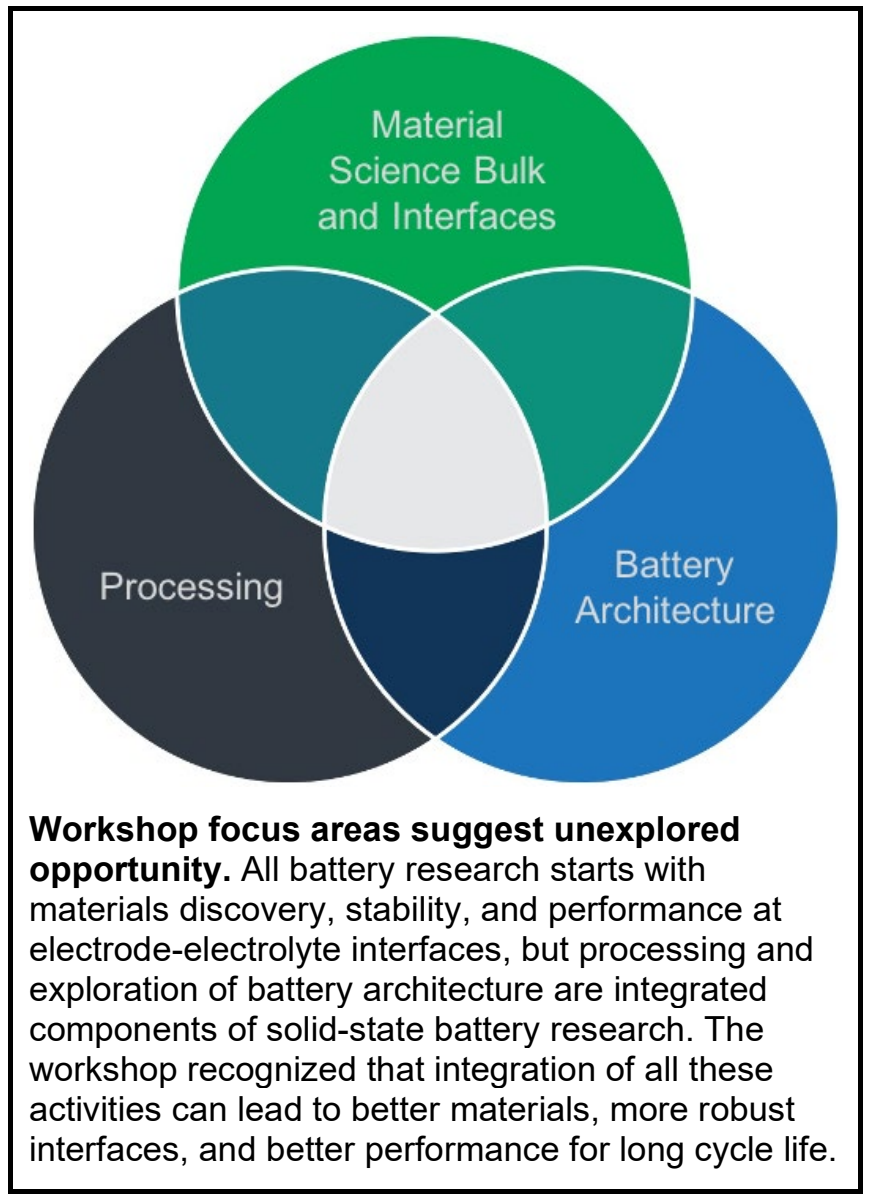

- In filling a porous ceramic electrolyte with a lithium nickel manganese cobalt oxide (NMC) cathode slurry, a waxy plastic crystal electrolyte accommodates changes in the cathode's volume during cycling. 
- Rethinking the original Monroe Newman model, with a different initial mechanical boundary condition, namely a flat solid electrolyte pressed onto a Li surface with a bump, relaxing the stress at the interface always favors Li deposition to the original valley. Consideration of elastic properties alone is insufficient to describe dendrite growth.

- Lithium phosphorous oxynitride (Lipon) amorphous thin films resist crack initiation by indentation at room temperature. This surprising property, combined with the earlier measures of creep, suggests that the ductility may be the reason that Lipon interfaces are robust with both cathodes and Li metal.

- Soft redox-active materials used as the active cathode component improve the electrolyte contacts.

- Glassy lithium thiophosphate is formed under pressure when heated above the glass transition temperature. Inclusion of Kevlar fibers reinforces the electrolyte and helps to blunt crack tips.

- Glassy electrolytes can be drawn to form thin flexible sheets at temperatures above the glass transition temperature and below temperatures for crystallization. Promising compositions open the temperature range and increase conductivity.

- Although the ionogel electrolyte is $75 \%$ molten salt, it is a solid after it undergoes a sol-gel reaction. The molten salt is contained in a silica matrix, yielding high conductivity at room temperature and wide electrochemical stability.

- Advanced methods to characterize buried interfaces during cycling are becoming available to complement electrochemical analysis. These tools link well to computational studies for greater insight into key mechanisms in solid-state batteries.

- Molecular dynamics (MD) simulations indicate that amorphous $\mathrm{Li}_{3 x} \mathrm{La}_{2 / 3-x} \mathrm{TiO}_{3}$ (LLTO) is stable in contact with Li metal. This finding supports the results of earlier experiments in which it was found that amorphous LLTO is stable but that the crystalline perovskite LLTO reacts with Li metal.

This report resulted from a review of the workshop by the organizers, with edits and additions from all participants. With contributions from all participants, this report represents a consensus of what is most important to consider for further investigations to enable low-cost, high-performance, long-lasting, and scalable solid-state batteries.

Solid-state battery science can be parsed by components, classes of materials and interfaces, physical and chemical processes, phases, lengths and temporal scales, processing, and performance. The body of this report is divided into three sections:

- Sect. 1,2, "The Science Gaps in Study of Materials and Interfaces"

- Sect. 1.3, "The Knowledge Gaps in the Science of Processing"

- Sect. 1.4, "Knowledge Gaps and Opportunities to Design Battery Architectures for Strength"

An outline of what is known and science gaps, organized by battery component, is found in Appendix C.

Although not stated as specific goals for the workshop, participants also addressed practical tradeoffs in manufacturing efficiency, materials costs, materials handling, and environmental sensitivity. Discussions touched on opportunities and barriers for US battery manufacturing. The need for standardization and statistical analysis ${ }^{1}$ was addressed as reported properties vary unacceptably among laboratories studying

${ }^{1}$ S. Randau et al., Nat. Energy 5, 259-270, 2020. 
nominally the same materials. Further, a careful safety evaluation is needed to replace the assumption that solid-state batteries are much safer than Li-ion batteries, where the industry has learned to manage the potential hazards effectively. A note from the playbook at Amat Inc. was shared and seems quite appropriate to our goals, "Think big, Test small, Fail fast, Learn quick."
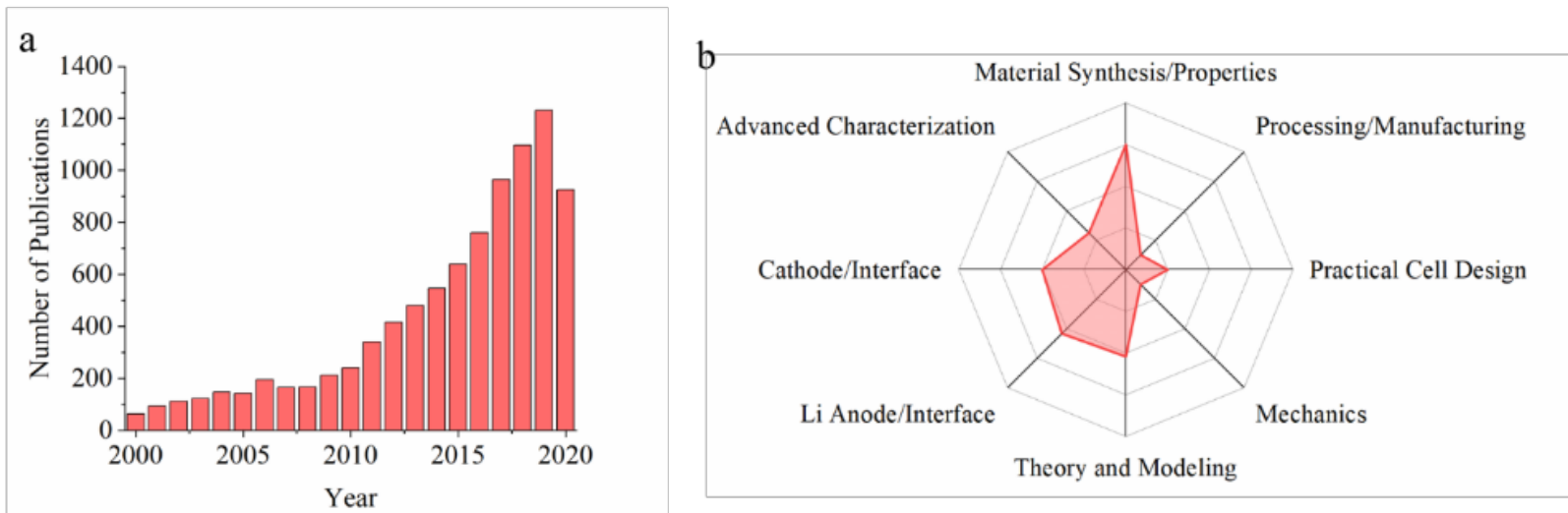

Analysis of publications and review articles for solid-state batteries. This literature analysis supports the intuition expressed by the workshop participants. There are important aspects of solid-state batteries that have received little attention. The images show (a) the number of peer-reviewed solid-state battery publications from 2000 to 2020 and (b) a radar plot that compares the level of activities in key technical areas for solid-state batteries based on analysis of 12 recent review articles [1-12]. For each of the references analyzed for the radar plot, we determined the approximate amounts of text and the number of references directed to each research activity. This was averaged for the 12 review papers; the plot indicates the average.

1. Y. Li et al., Small Methods, 2000111, 2020.

2. S. Tang et al., Adv. Energy Mater., 2000802, 2020.

3. S. Randau et al., Nat. Energy 5, 259-270, 2020.

4. H. Liu et al., ACS Energy Lett. 5, 833-843, 2020.

5. Q. Zhao et al., Nat. Rev. Mater. 5, 229-252, 2020.

6. L. Xu et al., Adv. Energy Mater., 2000648, 2020.

7. L. R. Mangani and C. Villevieille, J. Mater. Chem. A, 8, 10150-10167, 2020.

8. J. Liu et al., Nat. Energy 4, 180-186, 2019.

9. M. Ghidiu et al., J. Mater. Chem. A 7, 17735-17753, 2019.

10. X. Wang et al., Adv. Mater.32, e1905219, 2020.

11. Y. Xiao et al., Nat. Rev. Mater. 5, 105-126, 2019.

12. S. Xia et al., Chem 5, 753-785, 2019.

A future workshop could address two topics that deserve more attention than they were given at the May 2020 workshop: (1) mechanical challenges of solid-state batteries and (2) the science of processing materials for solid-state batteries. These topics deserve more attention than could be allotted at the May 2020 workshop and so the sections in this report are quite short. Yet, there are needs and opportunities for innovations that can transform the design and processing of solid-state batteries. The battery community has only recently engaged a larger number of experts in mechanical analysis of materials and structures, which is critical for systems that cannot rely on liquids to fill gaps opened during cycling. Similarly, engaging experts from broader processing fields may help identify new and unexplored manufacturing approaches with a more sophisticated understanding of the challenges.

To supplement these recommendations, a literature survey of solid-state batteries was conducted at Oak Ridge National Laboratory (ORNL). The volume of literature has grown and rate of publication has 
increased over the last decade. To ensure a representative perspective for this report, a dozen recent review articles were analyzed based on their emphasis of key technical areas for solid-state battery development. The analysis revealed that significant progress has been made in new materials discovery, but integration of these materials into practical devices has been hindered, in part perhaps, due to underemphasis of processing science and solid-state mechanics. Overall, this finding is consistent with our recommendation for a follow-up workshop focusing on these key topics.

\subsection{THE SCIENCE GAPS IN STUDY OF MATERIALS AND INTERFACES}

Progress on solid-state batteries typically surges following the discovery of a new and promising solid electrolyte. So far however, every known solid electrolyte has one or more drawbacks that must be overcome for application in a functional battery. Work should continue to discover new promising electrolyte materials through experiment and computation, with the expectation that there is indeed a material able to meet almost all goals (e.g., wide voltage stability window, sufficient ionic conductivity, low electronic conductivity, good mechanical properties). However, even if the ideal electrolyte was found, development of the other battery components (the active cathode and Li metal anode as well as their interfaces with the solid electrolytes) would need a strong focused effort. A clear understanding of the challenges to integrate components into full batteries will inform the search for new materials. These issues dominated discussion at the workshop more than the search for an ideal solid electrolyte.

The following questions need to be answered to fill the science gaps that exist in the development of an optimized Li metal anode:

- What defect generation/annihilation processes operate in Li films $(<30 \mu \mathrm{m}$ thick $)$ when Li is plated and stripped through a generic solid electrolyte?

- What conditions (such as rate, temperature, stress, duty cycle history) modify the plating and stripping behavior of Li?

- What are the stress relaxation mechanisms for Li, and how do they change with type and magnitude of the stress field?

- How do Li properties change with impurities or alloy elements?

- Is a seed layer of Li needed to template the plated Li?

- How do interphase regions, formed by reactions or additions at the solid electrolyte/electrode interface, govern transport at solid electrolyte/electrode interfaces?

The Li metal anode is common to all the batteries considered at the workshop, yet this component may be the least studied. We tend to ignore what seems at the outset to be the material requiring the least attention due to its malleable nature. In the last several years, Li metal has captured more attention by the US Department of Energy (DOE) Vehicle Technologies Office and the Advanced Research Projects Agency-Energy (ARPA-E), and there was considerable discussion among participants on this topic. "We know so much more now than just 5 years ago, but we are just getting started," reported Paul Albertus. One key finding, by nanoindentation and compression of Li micropillars, is that when the stress-volume in $\mathrm{Li}$ is small, the hardness and yield strength can be much larger than typical behavior for bulk Li. Consequently, we need to determine the relevant length scale for mechanical tests to inform our understanding of the $\mathrm{Li}$ anode and the mechanisms leading to Li redistribution, particularly when related to battery failure. 
For efficient Li utilization to meet goals for high energy density, managing the amount of Li to balance the cathode capacity is the key challenge. Li must remain dense and in intimate contact with the solid electrolyte, cycling with $\geq 99.98 \%$ coulombic efficiency, and minimal redistribution on any length scale for long battery life. For a "lithiumfree" design, all the Li that participates in the electrochemical reaction initially comes from a Li-saturated cathode. Such a design approach might be a pathway toward higher energy density. Effective management of the Li inventory to achieve long life has been demonstrated in a few examples; e.g., thinfilm batteries with the Lipon electrolyte; polymer batteries with high-modulus SEEO $^{\mathrm{TM}}$ (SEO), a styrene and ethylene oxide block copolymer; and when $\mathrm{Li}$ is confined in $3 \mathrm{D}$ pores of a lithium lanthanum zirconium oxide (LLZO) ceramic electrolyte. However, even for the batteries that can support extended cycling, the Li clearly evolves. Immediate failures occur for $\mathrm{Li} / \mathrm{Li}$ cells when a high current density causes flaws along the interface, even for initially clean and smooth surfaces. Li filaments form

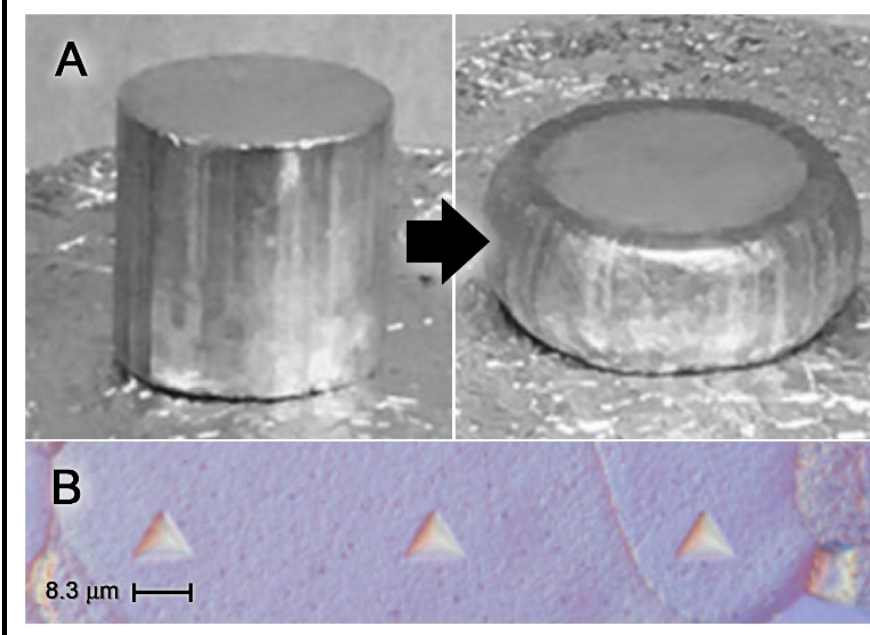

A matter of scale. Deformation of Li metal under compression depends on scale. (a) $A 1 / 2$ in. bulk cylinder deforms plastically under a 0.5 to $2 \mathrm{MPa}$ load. Depending on size, deformation of Li whiskers in compression (not shown) may require 10 to $100 \mathrm{MPa}$. (b) Upon nanoindentation to depths of $1.5 \mu \mathrm{m}$, the supported load (hardness) depends on the strain rate and displacement and approaches $100 \mathrm{MPa}$. (Indents are at $52 \mu \mathrm{m}$ spacing.)

A. Masias et al., J. Mater. Sci. 54, 2585-2600, 2019.

E. G. Herbert et al., J. Mater. Res. 33 (10), 1361, 2018.

rapidly through the solid electrolyte upon plating, and alternatively, interfacial voids form during stripping. For full cells with electrolytes based on $\mathrm{Li}_{3} \mathrm{PS}_{4}$ (LPS), the Li similarly roughens or fills cracks, depending on the particulate or glassy nature of the electrolytes, the current density, and the stack pressure.

Interesting discussions focused on Li plating and stripping as separate processes that need to be distinguished using a reference electrode or without reversing the current. Studies could also benefit by additional operando methods to characterize the dynamic chemistry and morphology of the buried interface between Li and a solid electrolyte (SE). Recent work has shown the utility of in situ and operando x-ray imaging for investigating Li interface dynamics, with much more to learn from other probes (neutron and sonic) that can see the buried interface.

For Li plating, important points from discussion include several advanced and refined models, which show surprisingly different results, depending on the initial state assumed for the Li-SE interface. Pressing a flat solid electrolyte against a Li surface with a bump, rather than applying a prescribed displacement, leads to predictions that Li plated at the interface will always tend to fill valleys. This is in striking contrast to the classic Monroe Newman model, and we believe highlights the importance of Li anode fabrication, at least for the initial cycling behavior. Additional electrochemical and mechanical studies are needed to draw relevant conclusions. Several new publications address the stresses that are created from localized nonuniform Li deposition at, or within, flaws of the solid electrolyte. The subtle effect of this stress on the plating overpotential is also reported in recent work. Earlier works, supported by DOE Office of Energy Efficiency and Renewable Energy Advanced Battery Materials Research program, address the interface formation and highlight the importance of various methods to clean and 
coat the solid electrolyte surface to improve wetting and adhesion of $\mathrm{Li}$ and to prevent or forestall $\mathrm{Li}$ filament growth.

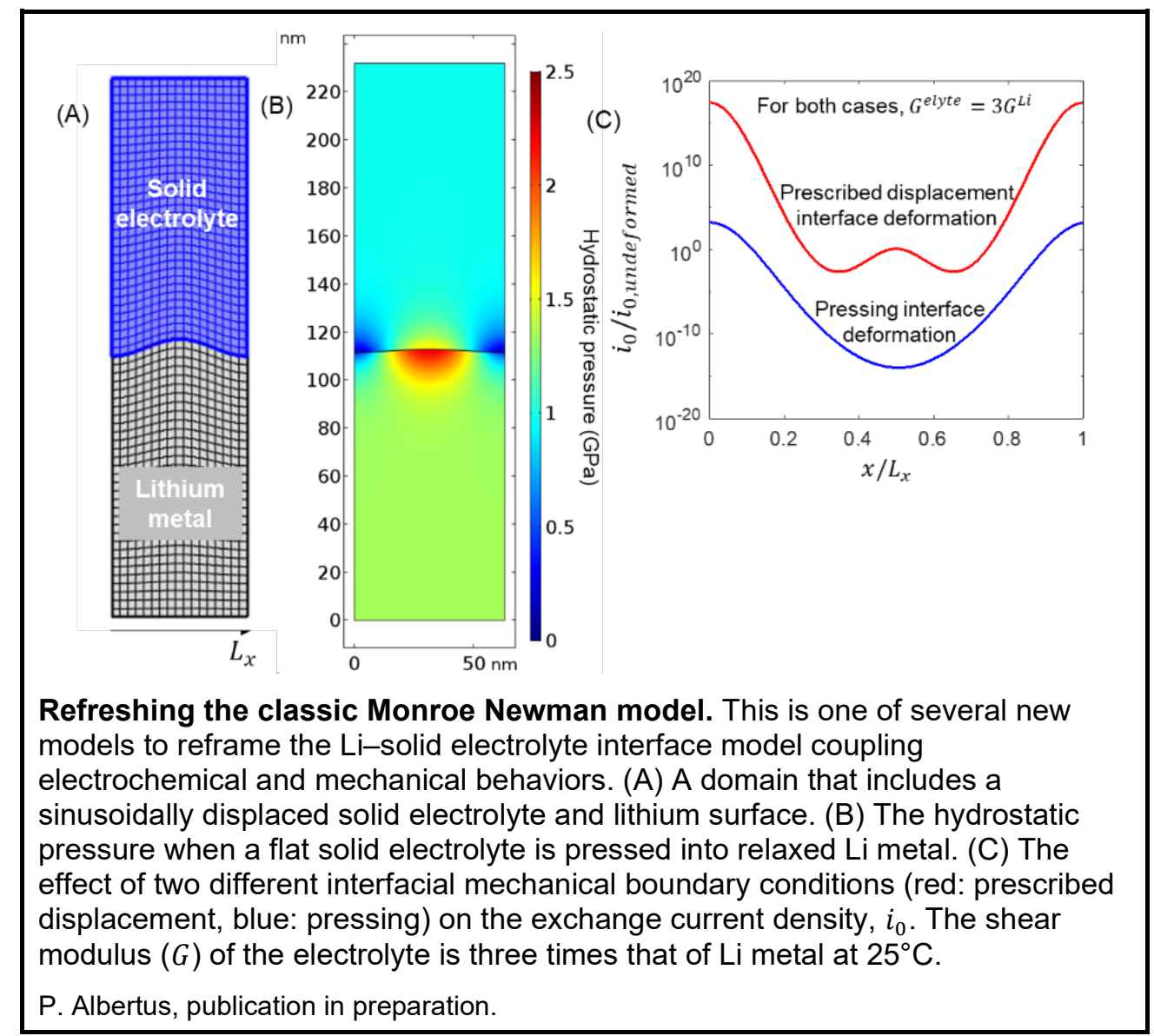

Then, upon stripping of Li from the anode through a solid electrolyte, experiments show that increasing interfacial resistance can be alleviated by an applied external stack pressure. Analysis leads to models where $\mathrm{Li}$ voids accumulate at the interface, and stack pressures of a few tens of megapascals, well above stack pressures for Li-ion cells $(0.03 \mathrm{MPa})$, may be needed for Li creep to match the stripping current and to maintain a good interface. In extreme cases, the Li peels away from the surface of the electrolyte. A fundamental unmet need is to have accurate creep maps for Li metal with clear identification and understanding of the prevailing mechanisms.

Based on the community's past results, future investigations need to intensify studies of Li behavior under continuous cycling using a carefully selected variety of duty cycles. Studies also need to move toward full cells and away from the symmetric Li/Li cells used in most studies. As observed for thin-film batteries, local redistribution of the Li depends on many factors, including rest periods in the duty cycle, and on the composition of the active cathode. Furthermore, there has been little investigation of the nature of the Li-free anode cycling where all or most of the $\mathrm{Li}$ is reformed upon charge.

\subsubsection{Science Gaps for the Solid Electrolyte in Contact with Li Metal}

- What promotes electrochemical stability or efficient passivation with Li? 
- What mechanisms are available to strengthen solid electrolyte properties, improve stability, and inhibit failures/fatigue over extended cycling with Li?

- How do the properties of the solid electrolyte and its interface, such current uniformity, modify the Li cycling?

- How does the Li anode "see" the cathode through a solid electrolyte separator?

Contact with Li metal and cycling also affect the solid electrolyte. This is the other side of the interface with its own unique challenges and science gaps. As with discussions on Li metal, the community has learned much about failure of solid electrolytes in recent years. Methods to improve any individual solid electrolyte remains a challenge. Studies have shown that (1) effective passivation of the interface reduces Li consumption, (2) a higher-modulus solid electrolyte formed with a dense smooth interface suffers less Li interface roughening or fracture due to protrusions filled or created by the Li, (3) a higher fracture toughness inhibits cracks that may form shorts, and (4) higher electronic resistivity slows internal local reduction of Li.

Several strategies to improve interface and bulk electrolyte performance were discussed.

Surface modification by coating to create a lithophilic and lithiophobic interface double layer may provide a means to direct where $\mathrm{Li}$ plates at the anode interface and avoid plating into cracks and flaws. The inherent smooth flaw-free surface of a glass is arguably the only way to create a sufficiently uniform interface for the $\mathrm{Li}$ anode, but further validation and novel ways to create thin coatings are needed to test that hypothesis. From a mechanics perspective, high yield strength and fracture toughness may be more general properties of amorphous inorganic electrolytes than

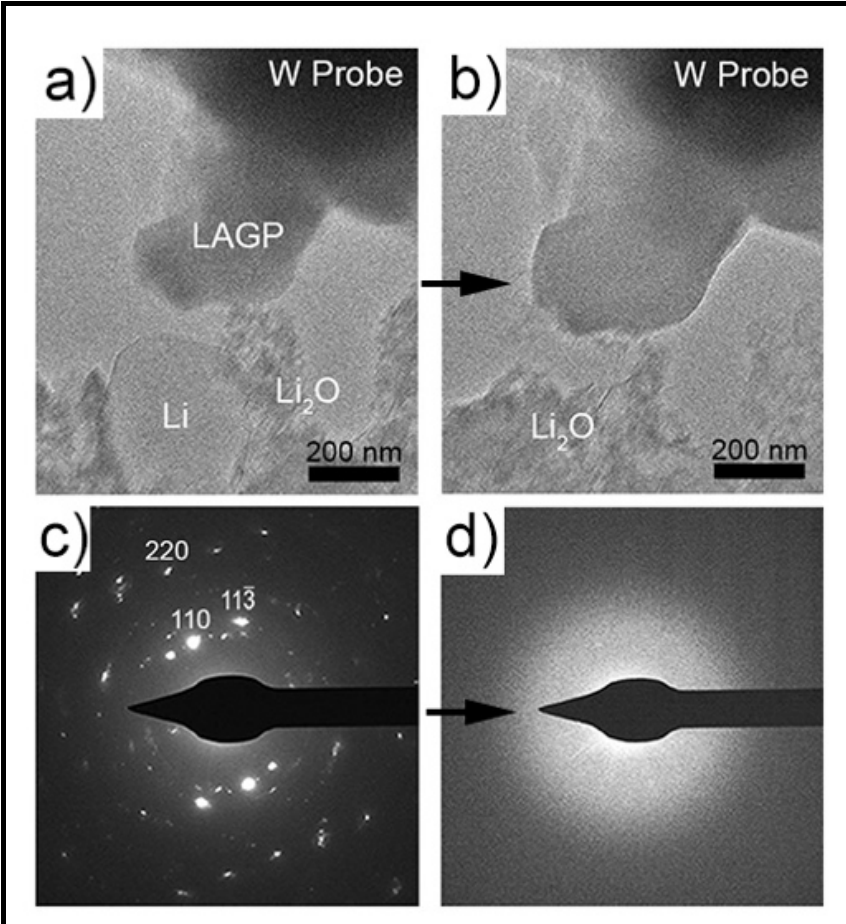

In situ transmission electron microscopy for a glimpse of $\mathrm{Li}$-solid electrolyte reactions, here with the LAGP electrolyte $\left(\mathrm{Li}_{1.4} \mathrm{Al}_{0.4} \mathrm{Ge}_{1.6}\left(\mathrm{PO}_{4}\right)_{3}\right)$ to probe interface instabilities and interphase formation under vacuum conditions. (a-b) This method has also been used with LLZO and Lipon. A single crystal of LAGP before (a) and after (b) reaction with lithium. (c,d) Associated diffraction patterns showing amorphization during interphase formation. At a buried interface, the extent of reaction may differ.

J. A. Lewis et al., ACS Energy Lett. 4(2), 591-599. recognized in earlier work, and amorphous inorganic materials offer a promising direction for discovery and processing of new electrolytes. In such materials, stress relief and recovery within small volumes may occur by shear and densification rather than by fracture. Other means to increase fracture toughness include the addition of fibers or particles; for example, additions of fine Kevlar fibers may blunt crack tips and reinforce electrolytes under stack pressures.

\subsubsection{Science Gaps for Active Cathode Materials and Solid-State Composite Cathodes}

- What can be achieved through defect and microstructure engineering to enhance reaction kinetics and mechanical properties for dense, single phase cathodes at all states of charge? 
- How can texture and grain structure be used to improve kinetics and reduce fracture?

- What are the relative pressure and electrochemical driving forces experienced by the active cathode material? Is there a restoring component that can provide more homogeneous reaction?

- What are the extensive design parameters for a resilient cathode-catholyte interface that maintains intimate contact through repeated cycles of different volume changes?

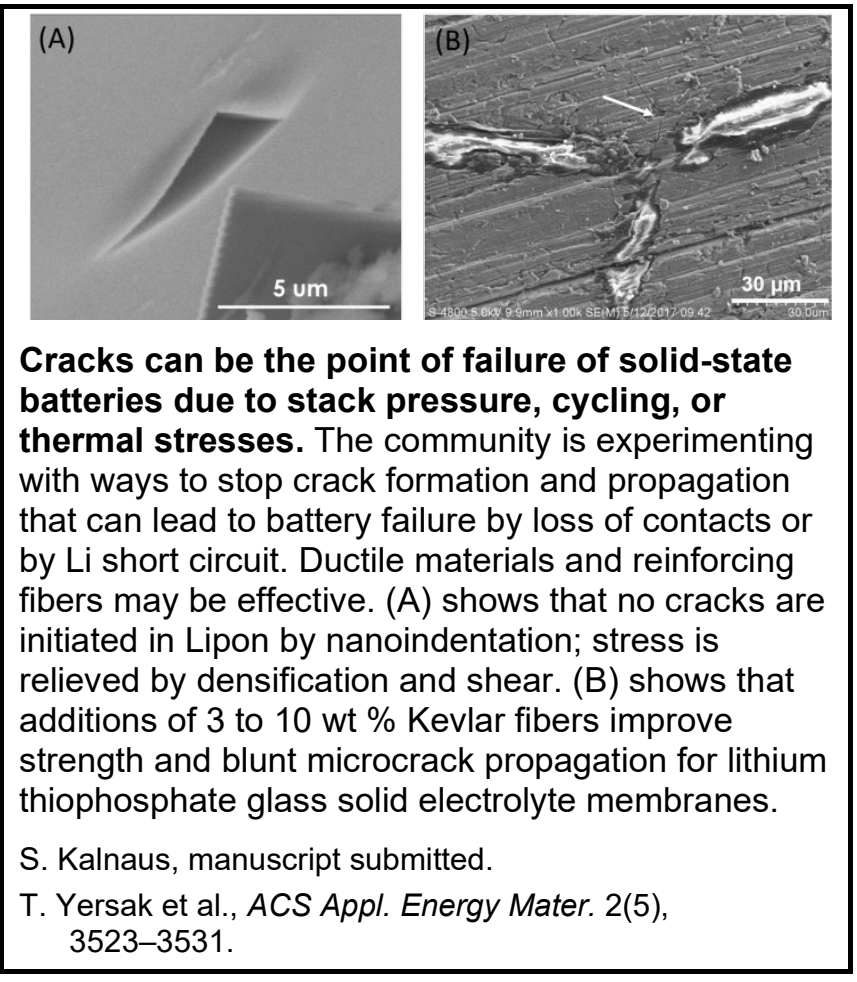

For the best energy density, the cathode must be the most voluminous component of the battery. If the cathode provides the mechanical support and battery substrate, then the current collectors, separator, and $\mathrm{Li}$ anode can all be applied as thin coatings with limited volume, weight, and cost. In traditional powder-cast cathodes, organic binders may suffice to form freestanding cathode membranes, or polymer electrolytes can be added to fill voids and improve transport. Often a stack pressure is required to maintain intimate and lowresistance contacts in the cathode composite. For a dry cathode that is mechanically stronger, the materials may be bonded, fused, or sintered. This complicates the processing but ensures mechanically robust solid-solid interfaces and grain boundaries. The key is to fabricate a cathode that will withstand the electrochemical cycling and that will provide facile electronic and ionic transport without the need to maintain stack pressure, which would add cost and weight.

Use of dense sintered membranes or films of the active cathode material is an option that should receive more attention. In practice it is rare to use a dense active cathode membrane because poor reaction and transport kinetics limit the effective cathode thickness; however, with better understanding of the ratelimiting process as a function of the state of charge, the kinetics may be improved. Refining the composition, defect structures, and microstructures through doping and processing can improve the kinetics and facilitate the use of dense cathodes. Defect equilibria analysis has proven useful for solid oxide fuel cell applications, where the defects, transport, and other physical properties of the electrolytes have been mapped and tuned. Attempts to analyze Li compounds with similar models are rare. Efforts to investigate doping, lattice substitutions, and stoichiometry may identify paths to enhance kinetics and reduce volume changes. Mechanical pressure is an additional variable that needs to be included in the defect equilibria analysis. This may generate a restoring force to partially alleviate stress in the active cathode or at the interface. This level of sophistication in the defect chemistry for intercalation reactions has not been necessary for Li-ion cathodes, which have much shorter diffusion path length than what is needed for solid-state batteries.

Cathodes formed as a composite present a near-term solution with added components to help manage the bonding and transport. The added phases may be a solid catholyte, an electronic conductor, or a solid mixed ionic-electronic conductor; the interfaces between these phases must be electrochemically stable, have low resistance for ion and charge transport, and survive repeated cycles with volume changes of the 
active material. Cathodes with reasonable cycling have been prepared with oxide or phosphate materials $\left(\mathrm{LiCoO}_{2}\right.$ [LCO], NMC, $\mathrm{LiFePO}_{4}$ [LFP], coated in some cases) mixed with carbons and either thiophosphate or polymer electrolytes. Generally, ceramic cathode particles embedded with a soft electrolyte function better using stack pressure to preserve interface contacts and/or when cycled at elevated temperature for faster kinetics.

Compared to cathodes for Li-ion batteries, even with soft catholytes, these materials cannot "heal" as they do in liquid electrolyte cells because the catholytes do not readily fill narrow spaces opened by fracture or phase separation. While it seems that the combination of soft and hard materials may provide the most uniform and compliant contacts, the interface between similar materials e.g., oxide/oxide) is believed to be intrinsically more stable with low interfacial resistance. However, it is difficult to fabricate a structure in which two hard ceramic materials are in intimate contact with each other.

Participants offered comments on their experience and expectations for promising

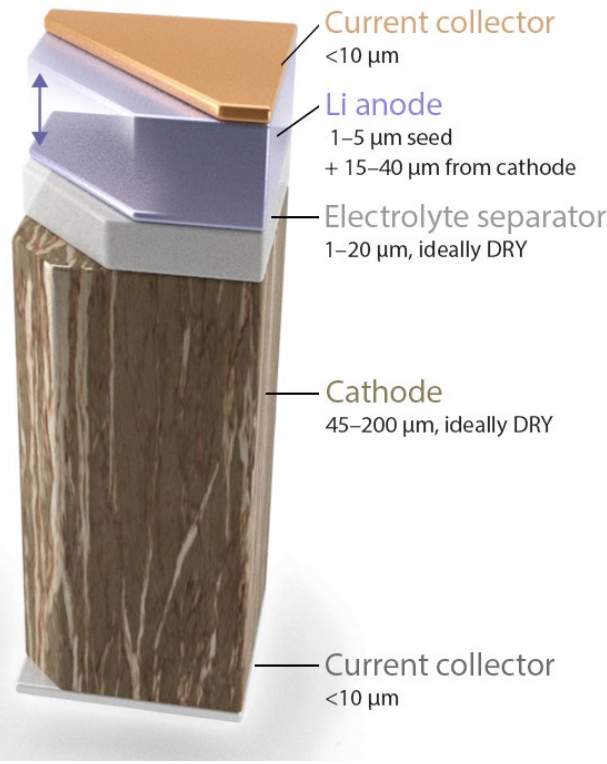

High-energy-density solid state battery stack. An ideal battery will maximize the volume of the cathode and minimize all other components, including the electrolyte and current collectors. For efficient utilization of the $\mathrm{Li}$ metal anode, most of the $\mathrm{Li}$ should be cycled for deep charges (indicated by the arrow). Development of scalable materials and processes to achieve these goals is critical for solidstate batteries to gain widespread adoption. cathode development. The use of waxy plastic crystal electrolytes provides an additional soft catholyte able to accommodate the strain associated with cathode volume changes. This has been demonstrated for slurry cathodes filling a 3D porous LLZO sintered structure. An interesting alternative is to identify and process soft organic cathode materials that provide good contact with hard solid electrolytes. How volume changes for a thick cathode membrane accumulate and add up to volume changes at the full cell level is still unexplored. Researchers and industry developing practical sulfide-based solid-state batteries find that appreciable external stack pressure is needed to maintain the contacts during cycling. Stack pressure must be tuned to improve contact without breaking the membranes; experimental housings to maintain the load for cycle tests are massive. Discussion ventured to the possibility of cleverly designed composites in which the internal pressure develops during cycling where and when needed to maintain the interface contacts.

With the many-faceted challenge to provide an adequate solid-state cathode, the list of materials science and processing science gaps is long, and there is urgency to get cathode membranes that are good enough to allow investigation of Li plating and stripping in full cells to proceed. There are examples where cycling of the anode and cathode, even with a thick solid electrolyte, are not independent. Experimental validation of the Li metal anode and solid electrolyte performance requires a full cell with a cathode that can deliver acceptable capacity and current at room temperature.

With the thick cathodes, it is critical to characterize the buried interfaces during cycling. Advanced characterization tools provide informative views about the structure, composition, and electrochemical 


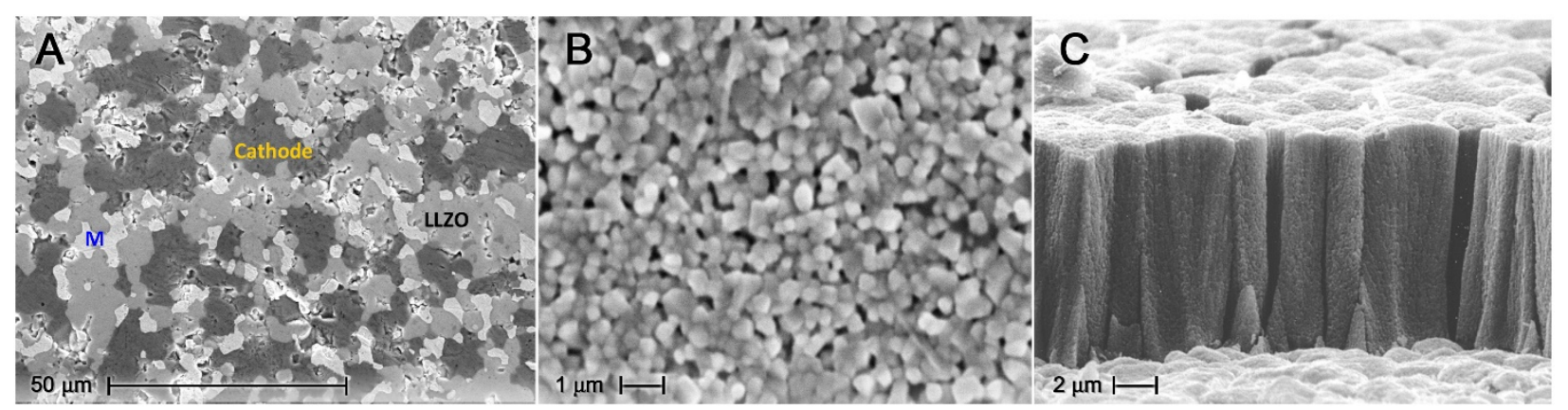

Solid-state cathodes. Whether a battery cathode is composed of the active phase alone or is a composite, its fabrication and design present opportunities to optimize the energy density, transport rates, and mechanisms to relieve stress from cycling. (A) $\mathrm{LiCoO}_{2}$ composite reported by Sakamoto (unpublished) was hot-pressed with LLZO, active cathode, and sintering aid. (B) Sintered LCO cathode reported by Dudney (unpublished) was prepared from powder by cold pressing and sintering. (C) LCO cathode grown by sputter deposition followed by anneal at $800^{\circ} \mathrm{C}$.

potentials at the buried electrode-electrolyte interfaces. These investigations are also valuable because they link very well to computational studies that provide greater insight to interfacial processes.

Operando studies are contemplated with advanced techniques, including scanning electrochemical cell microscopy (SECCM) and Kelvin probe force microscopy (KPFM). The SECCM tools scan the surface with a fluid-filled probe, providing information on the interface beneath the solid electrolyte; KPFM is used primarily to scan across a focused ion beam machined cross section. Solid-solid interfaces are also probed with Raman mapping that can reveal the state of stress, micro- and nano-scale computed tomography to reveal the morphology of the buried interfaces, and various operando diffraction studies that can probe crystalline phase evolution during cycling. While electrochemical studies are quantitative and precise, they do not identify the reactions or morphology changes, which must be probed with advanced tools, and the field will benefit from continuing development of novel methods to see the buried interfaces.

\subsubsection{Science Gaps for Solid Electrolytes}

- What properties of an interface between two different classes of solid electrolytes promote the rapid exchange of Li ions?

- How does the insertion of a single-ion-conducting electrolyte barrier layer at cathodes containing a binary electrolyte affect the battery polarization and resistance?

- What materials-strengthening mechanisms can be used for thin electrolyte membranes?

Using multiple different solid electrolytes in a battery imposes interfaces into the battery that are different from those of the solid electrolyte with an anode and a cathode. These differences may increase the design and processing options for providing stable and robust solid-state batteries and require further comment. Solid electrolyte properties have already been addressed in concerns about their stability in contact with dynamic Li and cathode materials. Many researchers have tried to form composites of multiple solid electrolytes, most often combining ceramic and polymer electrolytes, and it is apparent that $\mathrm{Li}^{+}$motion between phases can add an unacceptable resistance to the battery. 
The potential advantages of using multiple electrolytes in a solid-state battery are clear: (1) relieving the challenge to be stable with both metallic lithium and 5V cathodes, (2) balancing the tradeoffs of ion transport with mechanical properties, and (3) tailoring each electrode interface separately for robust contact and adhesion. In addition, inserting a single-ion-conducting electrolyte barrier can reduce the polarization within binary electrolytes. However, the structure, ion transport, polarization at interfaces between different solid electrolytes are not well understood, and the impact of employing multiple electrolytes needs to be clarified.

\subsection{THE KNOWLEDGE GAPS IN THE SCIENCE OF PROCESSING}

Unfortunately, due to lack of time, discussion to identify gaps in the science of processing was shorter than is needed to adequately address that important topic. A future workshop should include participants with varied insights to critically evaluate and identify processing opportunities. The organizers believe that processing science is important to moving the technology forward. It creates opportunities for new and modified materials that are not available with conventional processing methods. With many science gaps to be addressed to understand the materials and interfaces, manufacturing research is no doubt premature, but advanced materials processing could open new directions.

Several topics provide illustrative examples. Sintering a solid electrolyte into a dense or porous membrane and co-sintering to form the interface between the cathode and the solid electrolyte are necessary when simple cold compaction is inadequate. Bonding agents are proving useful to densify and fuse the dry cathode with solid electrolyte phases at lower temperature. Studies of rapid thermal heating, for example by radiative heating from a carbon

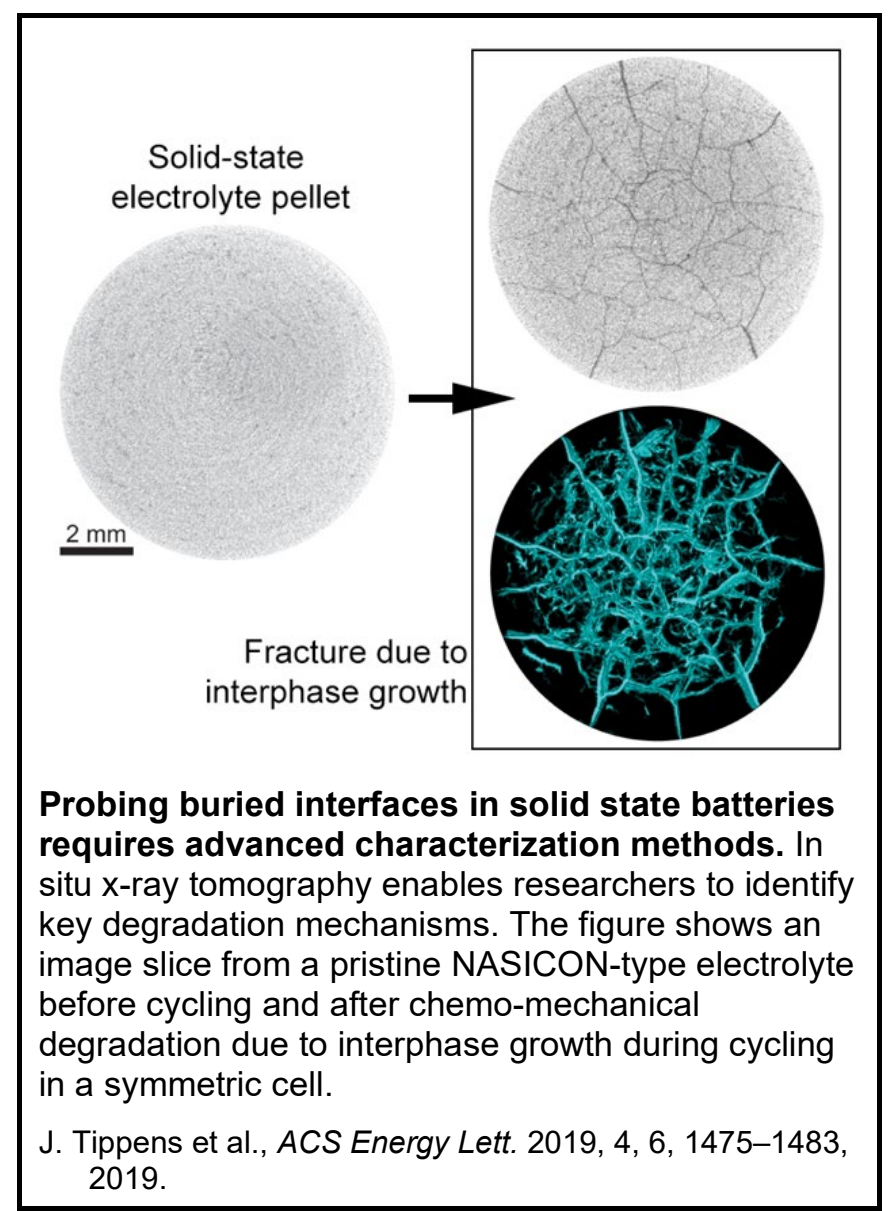
ribbon, ${ }^{2}$ may open new and practical processing directions. A science gap to address is to identify energy sources that effectively couple to wide bandgap electrolyte materials. Rather than by sintering, interfaces with a uniform contact area may be more easily achieved by filling or coating the interface with a liquid that is later solidified. For example, an ionogel can be solidified through a sol-gel reaction after a porous $3 \mathrm{D}$ cathode is filled with the liquid precursor. There are opportunities to investigate other novel and solvent-free processing routes to form intimate interface contact.

Processing determines the microstructure and material strength of the solid electrolytes used as the battery separator. There are well-known methods, such precipitation hardening, phase transformation toughening, and tempering used to strengthen structural ceramic and glass materials, but similar mechanisms are not reported for solid electrolytes. A science gap is to determine whether mechanisms exist to strengthen thin

${ }^{2}$ L. Hu et al., Science 368 (649001), 521-526, 2020. 
solid electrolytes and cathodes without impeding transport. Mechanisms that avoid addition of inactive components are particularly attractive because they do not affect weight or cost. Advantages of glass and amorphous electrolytes include chemical stability and ductility in addition to providing a naturally smooth surface. How to efficiently process thin amorphous materials by quenching will require new insight. We note that battery components are processed ideally as integrated coatings or composites, not as standalone parts.

The connection of processing innovation and the competitiveness of the US battery industry was also discussed. There is tension between short-term commercialization with existing equipment and a longterm horizon employing an investment in a totally new processing line. Looking at the next-generation batteries, a transformational battery enabled by a new processing line might give the United States the leverage and motivation to compete. Initial applications that are smaller and less demanding than EVs may provide important markets. All recognized that for commercialization, solid-state batteries must complete well on performance compared to advanced Li-ion technology, even if not at first on the price.

\subsection{KNOWLEDGE GAPS AND OPPORTUNITIES TO DESIGN BATTERY ARCHITECTURES FOR STRENGTH}

Most solid-state cells are, at present, a stacked design with the cathode composite borrowed from a typical Li-ion architecture. Exceptions are the 3D designs formed using 3D-templated cathodes, or more recently, 3D-porous solid electrolytes formed by freeze casting or burnout of sacrificial components. Another is a so-called "2.5D" design, which consists of a 2D Li-anode sheet with a 3D cathode plus solid electrolyte structure. These designs have been adopted to increase the interfacial area and thus to reduce local current density at the electrode-electrolyte contacts. That approach is promising, but in most cases there is not a clear pathway to cost-effective scale-up.

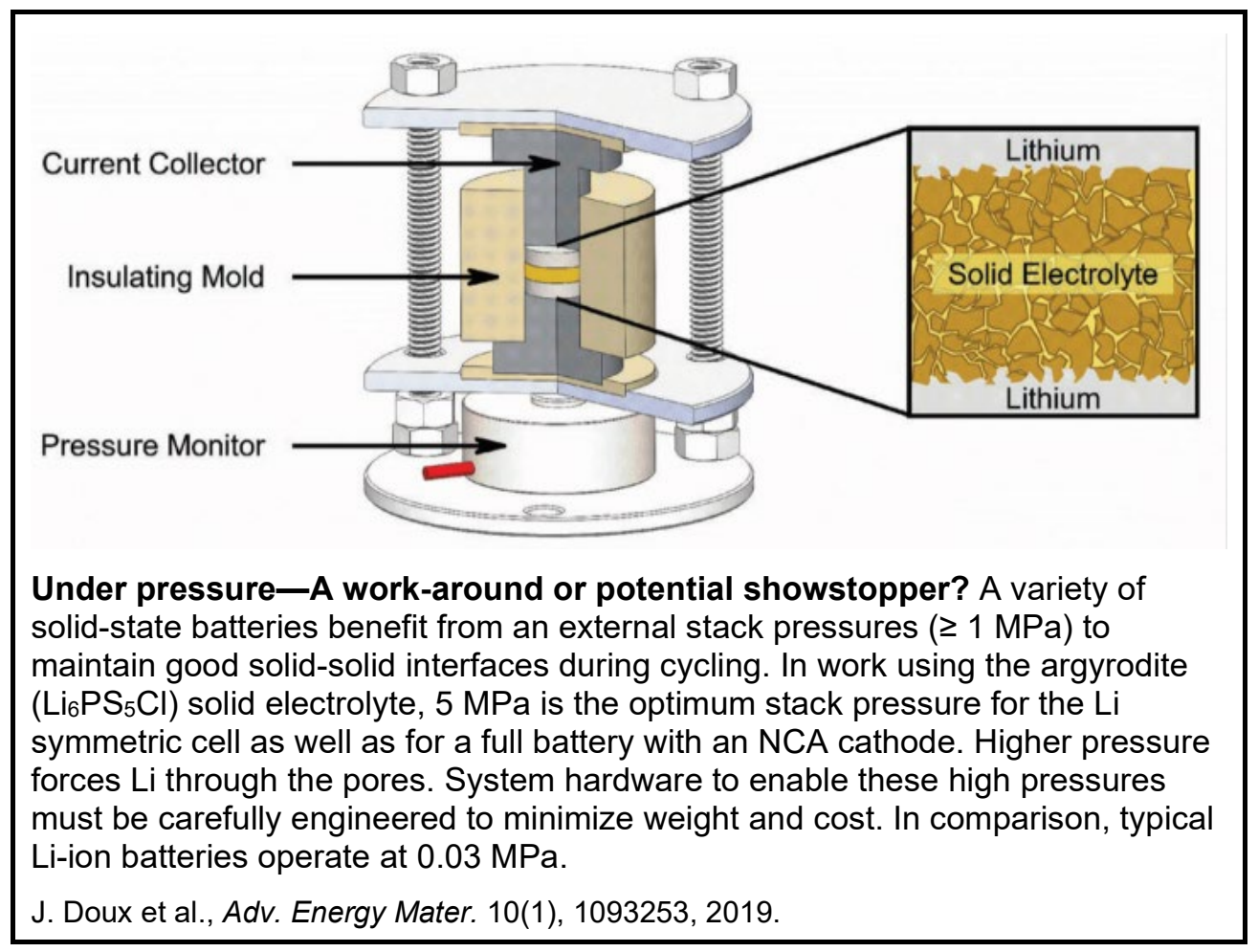

Alternative architectures may also enable mechanically robust structures and interfaces. Research in such structures is motivated by concerns that the cost of maintaining a stack pressure on the solid-state cells 
will be prohibitive. Discussion focused on how materials and composites should be processed and bonded for solid-state cathodes. Advanced manufacturing may make a variety of well-controlled architectures possible. Many interesting questions must be considered:

- Can volume changes be cleverly directed to provide internal compression at the material interfaces to replace large external battery stack pressures?

- Are there other ways to temper the cathode sheet and its internal interfaces to strengthen and resist fracture?

- What other particle and architecture engineering should be considered?

- Are there ways for the composites to maintain their shapes though extensive cycling and fluctuations in internal stresses?

- How does the inevitable volume change from plating and stripping the Li metal affect the solid-state battery and packaging?

\section{CONCLUSIONS}

\subsection{PATHWAYS TO SOLID-STATE BATTERIES-ADDRESSING SCIENCE GAPS}

The participants in the Solid-State Battery Workshop recognized three opportunities to address fundamental knowledge gaps that currently impede the development of solid-state batteries. Two address individual components and their interfaces ( $\mathrm{Li}$ anode and cathode), and the third is an accelerated path to integrating all the components for full cells (see Sects. 2.1.1-2.1.3). A future workshop may further inform recommendations for (1) expanding emphasis on the structural mechanics and chemo-mechanical coupling and (2) opportunities for novel processing to reveal new materials, new architecture, and improved interface properties.

\subsubsection{Study Control and Efficient Cycling of the Li Metal Anode}

The driver for solid-state batteries is the high energy density based on efficient cycling and confinement of the metallic Li anode. Significant improvement in efficient and extended cycling of Li metal resulted from the recent ARPA-E IONICS program, where Li/Li cycling helped identify several practical and manufacturable solid electrolytes for ongoing research. While the reasons for poor Li cycling with solid electrolytes are becoming clearer, the solutions are not. Application of large stack pressure is not a practical solution; if needed, it may be a showstopper for application of solid-state Li batteries in EVs. Alternative solutions will be informed by tackling the science gaps discussed at the workshop. Issues that will inform understanding of the Li metal anode include research to (1) compare the Li cycling performance of full cells versus Li/Li cells; (2) compare cycling at different temperatures, including where $\mathrm{Li}$ is molten; (3) assess the effects of various impurities in the $\mathrm{Li}$; and (4) compare Li from different sources.

The Li sources include commercially rolled and passivated ultrathin $\mathrm{Li}$, laboratory-vacuum-grown $\mathrm{Li}$ films, and Li grown electrochemically with a Li-free solid-state cell. Alternatively, the option to step back to use anodes of Li alloys or to provide a scaffold for Ag-coated carbon, as reported by Samsung, ${ }^{3}$ should not be ignored, although these solutions will sacrifice overall energy density. Having a larger variety of composite electrolytes available for building cells will accelerate tests for the Li. Furthermore, it cannot

\footnotetext{
${ }^{3}$ Y. Lee et al., Nat. Energy 5, 299-308, 2020.
} 
be overemphasized that the path to understanding is to use methods and cells designed to "fail fast" and "learn quickly." For the Li anode study, this means that both the metallic Li and the electrolyte should be very thin, with little excess capacity to either sustain side reactions or perpetuate interface roughening that will eventually lead to failure.

\subsubsection{Study to Advance Dry, Solid-State Cathodes and Composites}

The solid cathode component is key to performance that matches or exceeds that of the Li-ion batteries. For a battery with the highest energy density, the active cathode should occupy the largest fraction of the battery and as such the composite cathode should act as the physical support. This has been a critical roadblock for implementation of solid-state batteries, including batteries using each type of separator electrolyte material. In most designs the cathodes do not deliver sufficient energy at room temperature and at reasonable stack pressures. Hybrid designs using a standard Li-ion battery cathode with a t tiny amount of liquid or gel catholyte were tested, but they too had interface reactions and poor transport that limited performance.

Scientifically, the cathode study is also a foundational research topic because it requires solutions for many of the critical interface science challenges where progress is needed. Within the cathode component, the effects of the volume changes, interface integrity, and phase connectivity for facile ion and electron transport need to be addressed to achieve the current and energy delivery per active area. Studies on topics such as the defect equilibria with state of charge, performance of solid interfaces between different materials, processing of optimized composite architecture, stress and fatigue effects, and relaxation mechanisms to relieve stress will all provide insights that can be applied to other interfaces and properties throughout the solid-state Li battery. Furthermore, this topic must address the science gaps in materials, processing, and architecture.

For a successful solid-state cathode, experts in battery materials and solid-state electrochemistry will work closely with experts in materials mechanics and processing to minimize and relieve the stresses from cycling, identify mechanisms and architecture for strengthening the materials and interfaces, utilize materials and processing to form rapid and direct transport paths for ions and electrons that span the thickness of the cathode, and reduce or eliminate the need for external stack pressure by switching to a displacement constraint. To maximize the specific and volumetric energy density, the cathode should be the largest component and should provide the physical support. As mentioned for the Li study, this project should implement procedures to "test small" and "fail fast" so to advance scientific and technological knowledge of the solid cathode as quickly as possible. For the cathode, this fail-fast strategy encourages researchers to reveal the presence of any residual solvent, test at ambient temperature and pressure, and assess the highest specific capacity for cathodes, including all of the phases (e.g., active material, conductive additives, and catholyte). Progress can also be enhanced by the study of model cathode interfaces, adoption of aggressive cycling conditions, determination of chemical $/ \mathrm{mechanical}$ data for computation, and continued development of advanced characterization tools to probe the interfaces during and after cycling.

\subsubsection{Solid-State Battery Development with Rapid Integration of Electrode and Electrolyte}

Delivering a full solid-state battery that meet the performance, cost, and manufacturability necessary for an electric vehicle within a normal program cycle of 3 to 5 years, or even 10 years, is ambitious, particularly when the best pairing of solid electrolyte and battery chemistry is not obvious. The program would need to reflect the Battery500 in size and coordination. Targeting a less-demanding application in a smaller program, such as a consumer electronics battery, may lead to short-term successes but risks diverting attention from the most challenging problems for large-scale deployment of EV applications. 
The workshop participants recognized several paths forward. There are already strong scientific efforts underway that could be expanded to include greater emphasis on mechanical, transport and property measurements. It is worth creating efforts that include statistics and process control to determine an average cell performance rather than reporting the best cell; attention to battery failure and the causes; and large-scale, organized, and integrated data analysis. A challenge is how to get input from companies that have tremendous expertise and knowledge organized around the practical aspects of making devices.

A different approach that may prove effective is to integrate battery components from the outset of the program. A Laboratory Directed Research and Development program with this objective has been underway at ORNL for 1 year. Rather than spending time optimizing or even refining each component, half cells are being fabricated and cycled at the earliest possible time. The components that must work together, are developed together. In this way, the intrinsic stability and processes for integration are the first considerations. Coupling this with the "test small, fail fast, learn quickly" strategy may be a way to expedite the research and development to complement programs looking at individual solid-state battery components and interfaces. For an ambitious program to re-envision a solid-state battery with maximum energy density, this might also prove to be an approach to rapidly select the most promising class of materials in 2 years. Without a deliberate approach, a large program may be fractured, much the same as we have now, with multiple promising solid electrolytes and battery chemistries being pursed at once within individual programs.

Other reasons for a large integrated program and the keys for its success, as discussed at the workshop include having experienced technical associates to build and test the batteries, access to many processing tools and experienced operators, sharing of materials and standardized methods for rigorous comparisons, and the free sharing of failures and difficulties among the participants in addition to the successes shared publicly as journal publications.

\subsection{OUTLOOK}

Solid-state batteries are believed to provide advantages in performance, cost, and recycling for nextgeneration energy storage systems. Yet success is not assured, and particularly for transportation, there are possible impediments to development and acceptance of solid-state batteries, including poor control of materials and interface, processing challenges and cost, performance that does not show compelling advantage over advanced Li-ion technology, and high cost in maintaining stack pressure for solid-state battery packs.

In the past, the United States was an early inventor of high-energy-density batteries, such as Na-S systems; however, US manufacturers were not persuaded to take on the development and manufacturing of these batteries or the next generation of Li-ion batteries, leaving these markets to be filled by foreign companies. If our research activities identify novel solid-state battery materials and/or novel processing, US manufacturing might have another opportunity to lead for domestic manufacturing. 



\section{APPENDIX A. WORKSHOP PROGRAM}

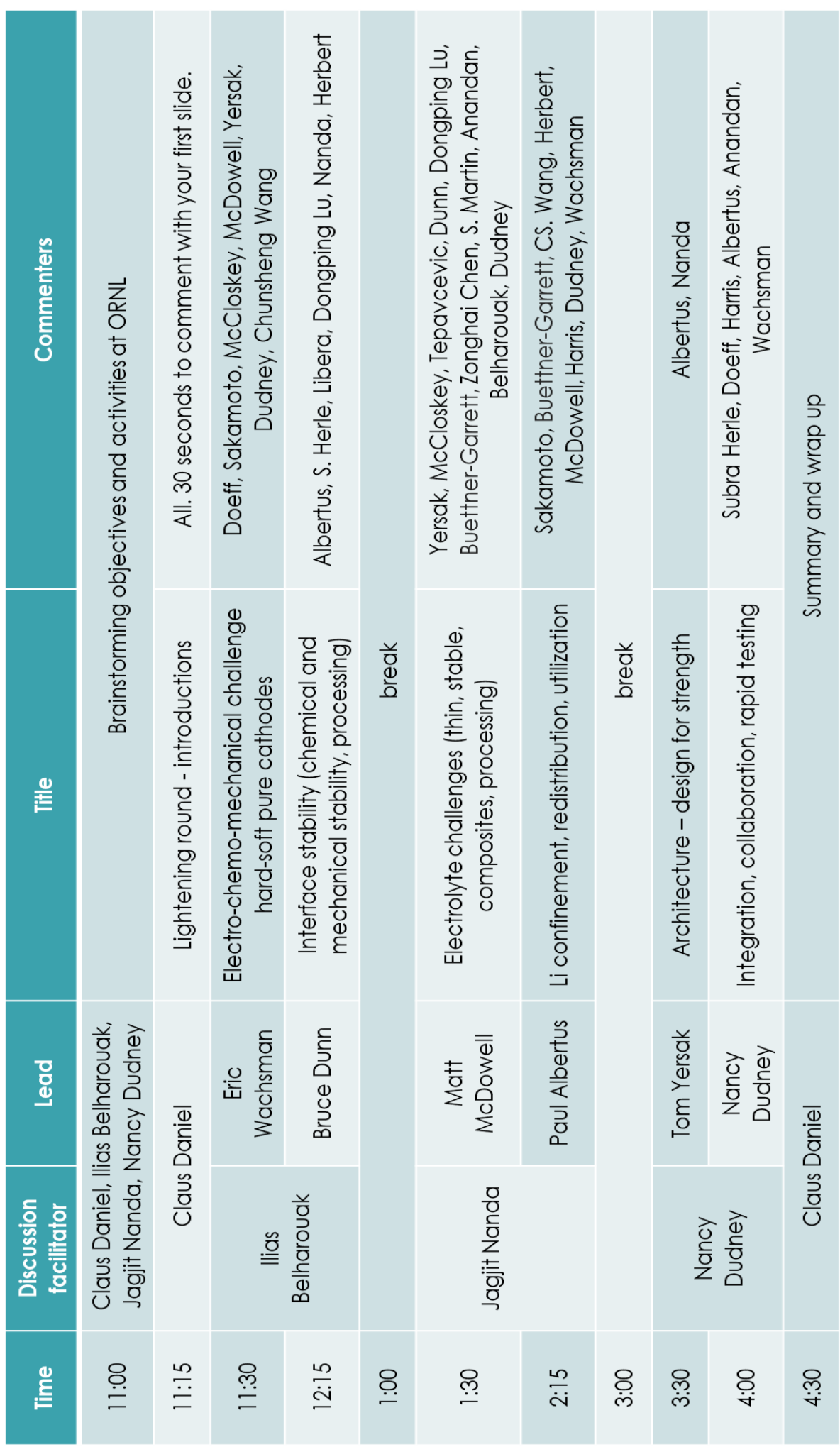





\title{
APPENDIX B. TWO SLIDES SUBMITTED BY EACH PARTICIPANT BEFORE WORKSHOP
}

\author{
Paul Albertus (University of Maryland)
}

\section{Albertus: Personal introduction and relation of activities to SSB}

Scope of program / researchers:

- Initiated and managed ARPA-E IONICS program focused on solid electrolytes for cycling Li metal. Through IONICS and other programs, oversaw $\sim \$ 50 \mathrm{M}$ of SSB projects at ARPA-E.

- Started as Assistant Professor at UMD in 2019, 3 students, focusing on Li metal cycling, looking at mechanics and electrochemical processes. Approaches include multi-physics continuum modeling and experiments on key mechanisms.

To read from my program:

- Perspective on cycling Li metal, including technical and economic metrics (i.e., when results are significant, and how to avoid cycling electrons and not $\mathrm{Li}^{+}$): Nature Energy 3, 16-21 (2018)

To read from the literature (I have a much longer list!):

- Best full cell, porous electrode, SSB results l've seen are from Kanno $(2016,2018)$. Shows potential of sulfide SE with $t_{+}=1$ to give high performance electrodes and reasonable energy density.

- Dendrite formation and propagation in oxide and sulfide SE: YM Chiang (2017), Sakamoto \& Dasgupta (2020).

- Importance of LiPON for thinking about Li metal cycling: Dudney (several works)
Unique capabilities, knowledge, resources:

- Strong knowledge and capabilities in continuum modeling of the coupling of transport, thermodynamic, kinetic, mechanical, etc processes on mechanisms of interest.

Experience at ARPA-E gave me a big-picture and a detailed perspective on SSB, including science to commercialization, current activities at US universities and companies, and DOE funding programs.

- A couple of points on making SSBs a commercial reality: (1) The US does comparatively little integrated scientific and industrial development in non-defense areas, and (2) In general, big companies in Asia are much more interested and willing to invest in SSB than big American companies, and are leading and will almost certainly lead the industrialization, if SSB turn out to be technically and economically worthwhile. The expected financial returns, time scales, and established industry in Asia make investments difficult to justify for the large strategic American companies that may have the capabilities to bring SSB to scale.

\section{Albertus: Critical science gaps and opportunities for SSB}

\section{High-level science gaps and opportunities:}

- Careful property measurements are important and more are needed. Mechanical properties, electrical properties, roundrobin measurements among different research labs of the same properties, measurements of how small compositional variations or impurities affect properties, and other measurement efforts could be expanded. This is improving

- There is a benefit in having standard materials and methods that can be shared among research groups to get statistically significant results. The present decentralized research structure with a large variety of material handling methods and no need for statistics to publish in good journals limits scientific progress. Companies do this.

Gaps in material science:

- Role of creep and plastic deformation of $\mathrm{Li}$ and the SE, and their influence on plating stability.

- More careful exploration of other metal electrode / SE systems for key insights into stability may be fruitful. A variety of metals such as $\mathrm{Ag}, \mathrm{Na}, \mathrm{K}, \mathrm{Cu}$ have a solid electrolyte that conducts the $\mathrm{M}^{+}$cation.

- The science of "anode free" cells including interface formation, cycling stability, role of defects, etc.

- Polymer electrolytes for a porous cathode.
Gaps in processing science: Gaps in battery architecture:

- Having a strong understanding of - "Anode free" cells may offer opportunities and how to manage and achieve challenges for battery architecture (tabbing?). stability using an interfacial coating - Volume accommodation for Li metal cycling is between two reactive materials is important. "3D" Li metal anodes as an example important. Do we know enough to of approaches to explore carefully. Volume say when an interlayer will result in accommodation over a range of length scales stability vs not? may be required (e.g., even tab location effects

- What purity, of SE, Li metal, etc, do on Li rearrangement may be important). we need, and how can it be achieved in a cost-effective manner?
- Novel ways to achieve high pressures in the applying the pressure through an external clamp. needed locations within a solid state cell without

Paul Albertus: albertus@umd.edu 


\section{Venkat Anandan (Ford Motor Company)}

- Personal Introduction

- Venkat Anandan- Leading a solid state battery (SSB) research group at Ford. His team is involved in the research and development of SSB technology for Ford electrified vehicles

\section{- Scope of my researchers/programs}

- We are working on oxides, sulfide, and polymers based SSB, and we are not inclined to any specific solid state technology

- Have several internal programs focused on solving some of the core issues in different types of SSB

- Ford has collaborations with several Universities and National labs

\section{- Unique Capabilities, Knowledge, and resources}

- Ford has a wide range of expertise and capabilities that includes but not limited to cell testing (coin cells to large format), design and development of modules and packs for electric vehicles, modeling capabilities, vehicle safety system design

- My team had published several patents in the area of SSB. Selected list as follows:

- Solid State Battery. US Patent 10530009.

- Composite separator with aligned particles. US Patent 9666852.

- Flexible composite solid polymer electrochemical membrane. US Patent 9478809

- Composition for sintered lithium titanate-lithium lanthanum titanium oxide composite. US Patent App. 16/036686

- Method for suppressing metal propagation in solid electrolyte. US20190006707A1

\section{Critical science gaps and opportunities for SSBs Venkat Anandan}

- Opportunity: SSB could provide high energy density along with enhanced safety compared to current Li-ion batteries. Since safety of current Li-ion battery packs are well managed in the EVs through engineering designs, we believe obtaining higher energy should be the primary focus for EV application. In addition, we also think significant improvement in rate performance and cycle life is needed for this technology. With that, we would like to highlight following challenges and gaps :

\section{- Challenges}

- Enabling Li metal anode with similar cycle and rate performance to Li-ion

- While enabling Li metal anodes, the research should also focus on eliminating excess Li or designing Li free cells. This approach could reduce the cell cost and complexity in manufacturing

- Higher discharge and charging rates are very critical to EV performance. Current SSB perform have poor rate performance, hence significant improvement in this area is required

- While manufacturing cells based on sulfides or polymers is relatively compatible with existing processes, manufacturing of oxide based SSB is still a challenge

- Gaps

- Though SSB are considered safer due to the use of non-flammable electrolyte, but there is insufficient data to validate the safety. More work is required to validate the safety of SSB

- Most of the SSB performance challenges are related to material and electrode interfaces. Unlike in Li-ion, the interfaces are not well understood in SSB. More research activity should be required to address this issue.

- Most of the solid electrolyte research so far focused on improving the ionic conductivity, since there are electrolytes with high conductivities, future research activity should emphasis on improving electrochemical stability

- Modeling and simulation techniques could play an important role in understand and designing new electrolyte and interfacial material

- New manufacturing processes and methods especially improvement in sintering of oxide materials are needed for the development of oxide based SSB 


\section{Ilias Belharouak (Oak Ridge National Laboratory)}

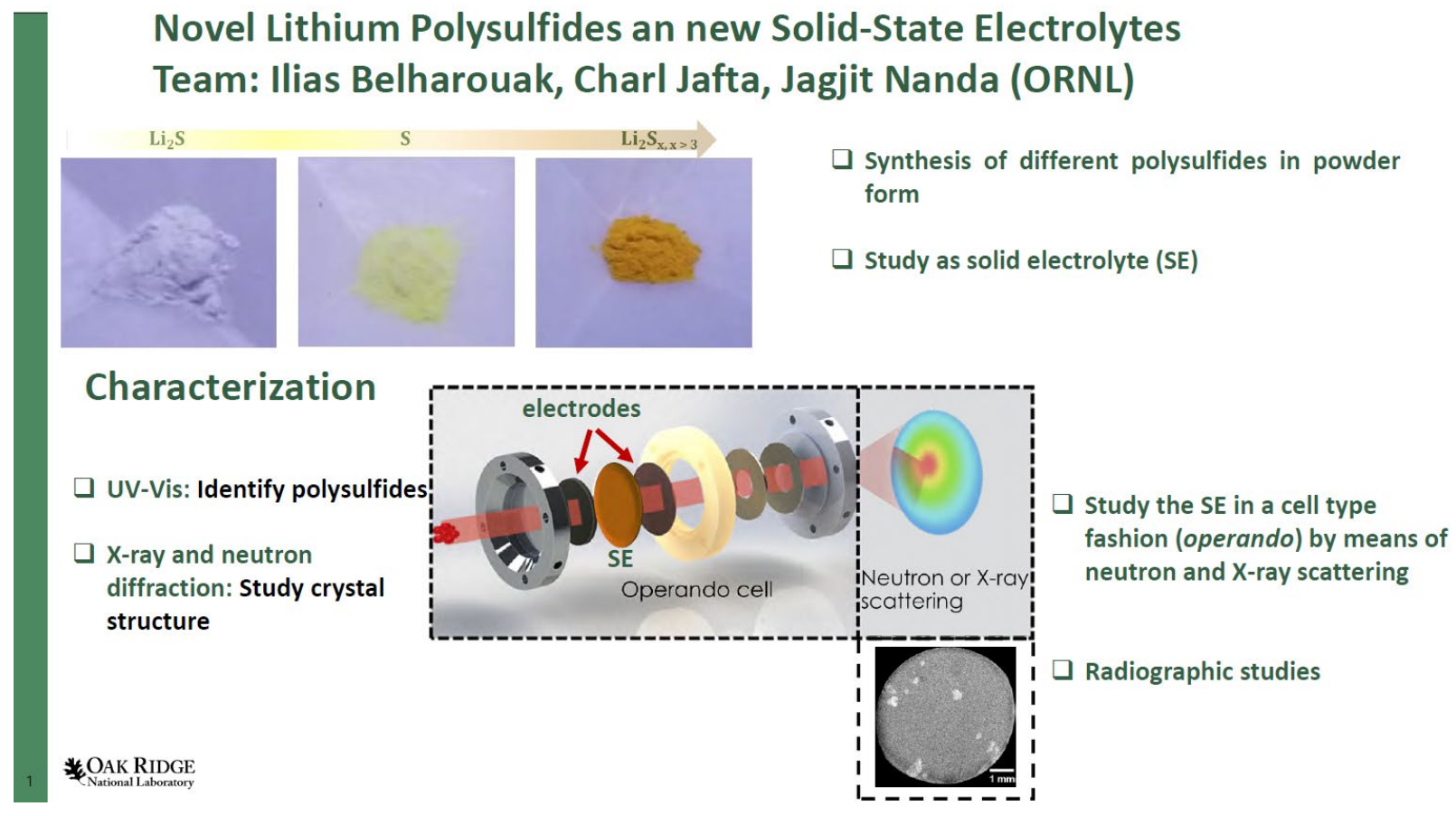




\section{Josh Buettner-Garrett (Solid Power)}

\section{Solid Power - Quick Facts}

- Located in Colorado with headcount of 56

- Solid-state batteries based on in-house developed and produced sulfide electrolytes

- Primarily focused on the electric vehicle market

- MWh scale pilot production line with equipment that mirrors traditional Li-ion infrastructure

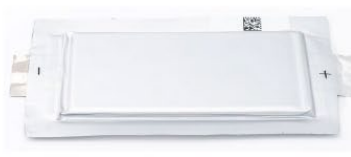

Pouch cells up to $20 \mathrm{Ah}$

(2 Ah shown)

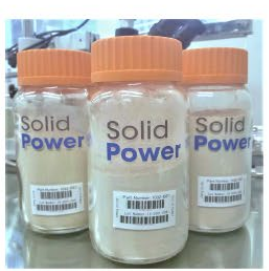

Broad collection of sulfide electrolytes

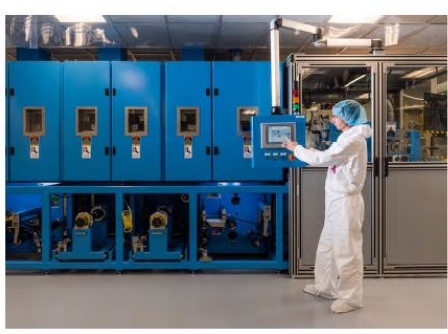

Pilot-scale slot die coating

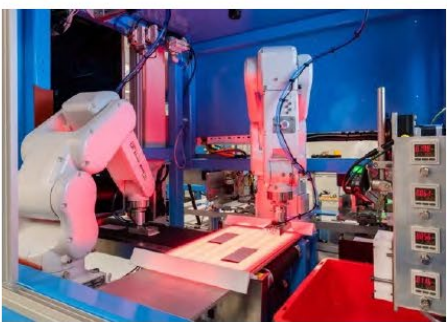

Automated electrode processing

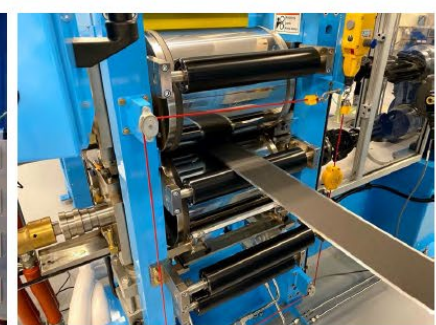

Roll-to-roll densification and lamination

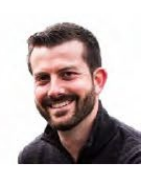

Josh Buettner-Garrett Сто

\section{Remaining gaps for Li metal solid-state cells with sulfide electrolytes}

Major remaining technical challenge for Solid Power is charge rate capability at low temperatures

Compatible binder/solvent systems!

- Existing binder and solvent candidates leave much to be desired in terms of conductivity suppression, adhesion, and slurry rheology

- Some binder-less or solvent-less routes look promising, but scalability and cost are questions

Better fundamental understanding of Li plating behavior and how to control

- Seems to already have an appropriate level of focus in the community 


\section{Zonghai Chen (Argonne National Laboratory)}

\section{Stabilizing solid/electrolyte interface from bottom up \\ Zonghai Chen (ANL)}

Common belief for cathodes:

Co-rich: detrimental phase transformation at $4.5 \mathrm{~V}$

Mn-rich: voltage fade above $4.5 \mathrm{~V}$

Ni-rich: increased chemical reactivity above $4.5 \mathrm{~V}$
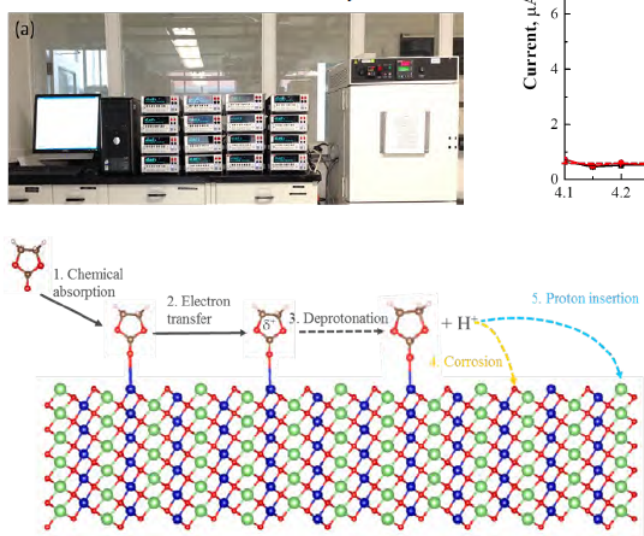

Bottom-up mitigation

> Poisoning the active sites on cathode materials

- Blocking electron transfer between the electrolyte and the cathode

- Improving the tolerance of cathode against proton

Ultimate solution (recent focus):

> Getting rid of liquid electrolytes: Solid-State Batteries (SSB)

> Technical lead of solid-state electrolyte processing lab

> PI of solid/solid interface diagnosis project (BMR)
Catalytic reaction mechanism: J. Phys. Chem. Lett., 2019, 10(3): 589-594 Electrochemical oxidation: J. Phys. Chem. Lett., 2017, 8(5): 1072

Chemical oxidation: ACS Appl. Mater. Interfaces, 2016, 8(5): 3446 Proton insertion: ACS Appl. Mater. Interfaces, 2020, 12(6): 7277 Mitigation:

ACS Appl. Mater. Interfaces, 2017, 9(51): 44542 ACS Appl. Mater. Interfaces, 2018, 1(5): 2254

Mater. Today Energy, 2018, 7:18

Chem. Mater., 2019, 31(8): 2723

Nature Energy, 2019, 4: 484

\section{Chemistry-process duplex is the key for realistic SSB R\&D Zonghai Chen}

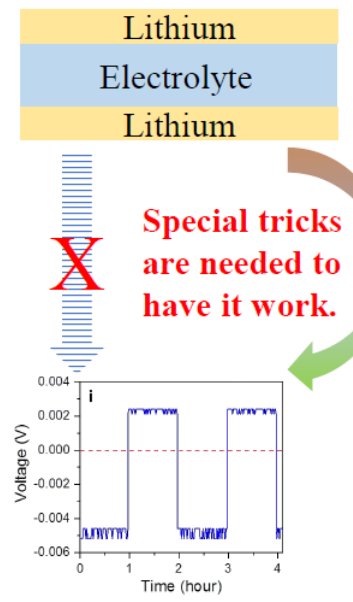

Ticks needed:

1) Building cells right after the material is synthesized.

2) Making thick electrolyte with aggressive polishing.

Both tricks are not practical for realistic applications.
Issues with aged LLZO powders (Al-LLZO, Ta-LLZO, Ga-LLZO)
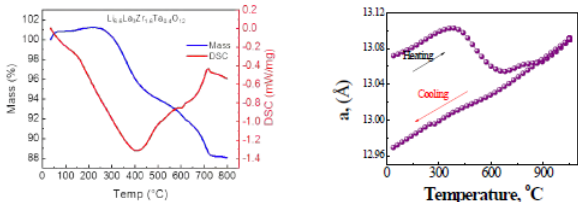

- Aged LLZO remains cubic phase, bot not the highly conductive phase we desired.

$>$ Significant $\mathrm{Li}-\mathrm{H}$ exchange occurs for aged sample.

$>$ High temperature processing is needed for recovering the desired structure.

Issues with hot-pressed LLZO (Ta-LLZO and Al-LLZO)
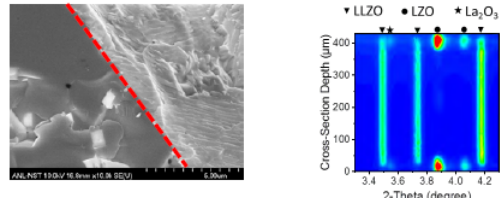

The interface layer after hot-press is dominated by $\mathrm{La}_{2} \mathrm{Zr}_{2} \mathrm{O}_{7}$ with a small amount of $\mathrm{La}_{2} \mathrm{O}_{3}$.

$>$ The central layer remains cubic LLZO.

- The decomposition mechanism at the interface is not clear yet.

Conclusion

$>$ The chemistry research cannot be decoupled from the process research

- The process (including synthesis and cell assembling) determines the exposure environment for solid-state electrolytes.

- A fundamental understanding on the interaction between electrolytes and the environment can provide critical guidance for rational design of chemistry-process duplex. 


\section{Marca Doeff (Lawrence Berkeley National Laboratory)}

\section{Who I am and what I do}

- Marca M. Doeff, Deputy Division Director Energy Storage and Distributed Resources Division, Lawrence Berkeley National Lab. Current chair of the Battery Division of the Electrochemical Society, Secretary-elect of Electrochemical Society

- Research Interests-materials for batteries including solid state batteries, Li-ion batteries, Na-ion batteries. Have worked on polymer batteries (in the 90s!) and LLZO.

- Some of my recent papers:

- "All-Solid-State Batteries Using Rationally Designed Garnet Electrolyte Frameworks" E. Yi, H. Shen, S. Heywood, J. Alvarado, D. Parkinson, G. Chen, S. W. Sofie, and M. M. Doeff ACS Appl. Energy Mater., DOI: 10.1021/acsaem.9b02101 (2020).

- "Oriented Porous LLZO 3D Structures Obtained by Freeze Casting for Battery Applications" H. Shen, E. Yi, M. Amores, L. Cheng, N. Tamura, D. Parkinson, G. Chen, K. Chen, and M. M. Doeff, J. Mater. Chem. A, DOI: 10.1039/C9TA06520B (2019).

- Scalable Freeze Tape Casting Fabrication and Pore Structure Analysis of 3D LLZO Solid-State Electrolytes" H. Shen, E. Yi, S. Heywood, D. Parkinson, G. Chen, N. Tamura, S. Sofie, K. Chen, and M. M. Doeff, ACS Appl. Mater. \& Interfaces, DOI: 10.1021/acsami.9b11780 (2019).

- "Solid State Electrolyte Considerations for Electric Vehicle Batteries" H. Shen, E. Yi, L. Cheng, M. Amores, G. Chen, S. Sofie and M. Doeff, Sust. Energy \& Fuels, $\underline{3}, 1647$ (2019).

- "Garnet Electrolyte Surface Degradation and Recovery", L. Cheng, M. Liu, A. Mehta, H. Xin, F. Lin, K. Persson, G. Chen, E. Crumlin, and M. Doeff, ACS Appl. Energy Mater., 1(12), 7244 (2018).

- Important recent papers from the literature:

- "A General Method To Synthesize and Sinter Bulk Ceramics in Seconds" Hu et al., Science $\underline{368}, 521$ (2020).

- "Benchmarking the Performance of All-Solid-State Lithium Batteries" Janek et al., Nature Energy $\underline{5}, 259$ (2020).

- "High-Energy Long-Cycling All-Solid-State Lithium Metal Batteries Enabled by Silver-Carbon Composite Anodes" Han et al. (Samsung) Nature Energy $\underline{5} 299$ (2020).

\section{Research Needs and Opportunities}

Marca M. Doeff

how do we get to real batteries?
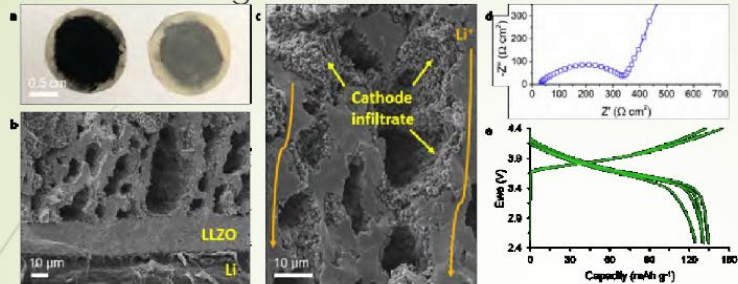

Cycle life/performance? Optimized structures and infiltration? Rapid sintering? Mechanical robustness?
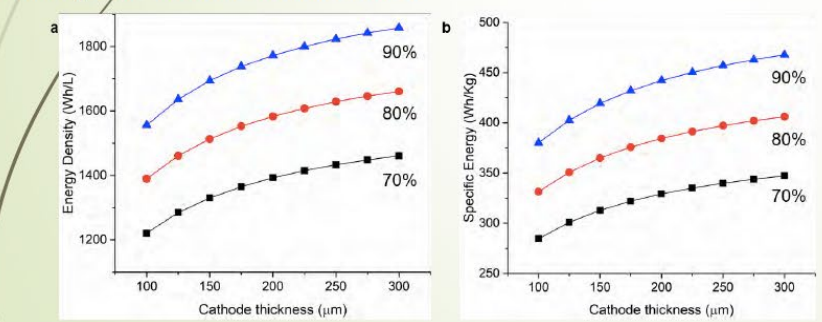

En ergy density vs. power density? Porosity vs. mechanical strength? Requires only $20 \%$ xs of Li, thin current collectors, $74 \%$ filling with NMC

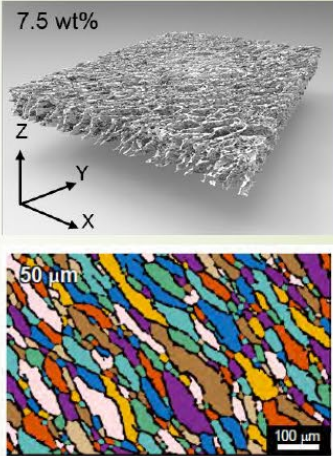

Optimum porosity?

Scalability?

Larger size cells-mechanical issues? Other designs (tubular, bipolar, etc.)? Other methods of making porous ceramics?

Other oxide electrolytes? Cost, especially sintering 


\section{Nancy Dudney (Oak Ridge National Laboratory)}

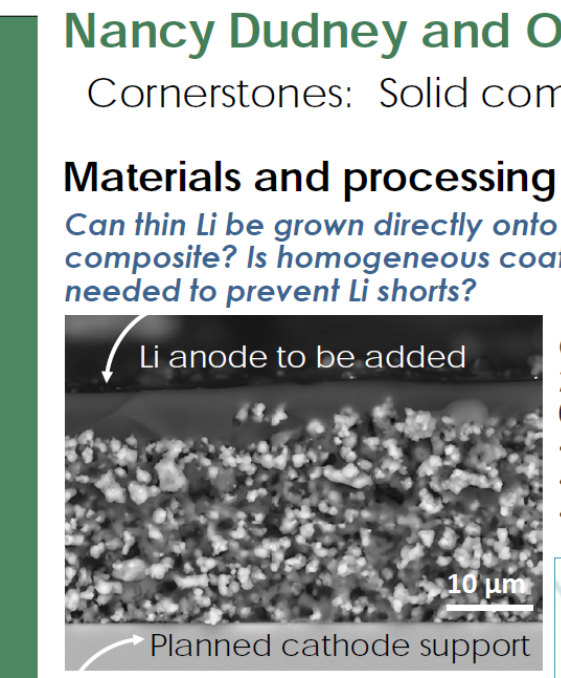

How to integrate fabrication of this composite electrolyte with cathode?

Composite electrolyte 20um thick $0.05 \mathrm{mS} / \mathrm{cm}(\mathrm{RT})$

- Ceramics, glasses (A. Westover) Polymers, single ion (X. Chen) Models, mechanics (S. Kalnaus)

Arbitrarily Thick Cathodes (LiCoO2) at $\mathbf{1 5}$ microns or more

N OAK RIDGE

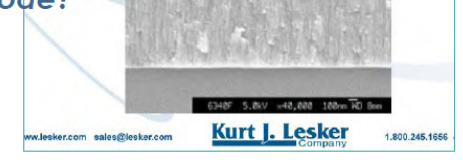

\section{Characterization}

- Neutron studies of interfaces and materials synthesis (R. Sacci, G. Veith)

- Other interface tools: Dynamic impedance precision coulometry, TOF SIMS,

- Mechanics tools: including nano- and picoindenters.
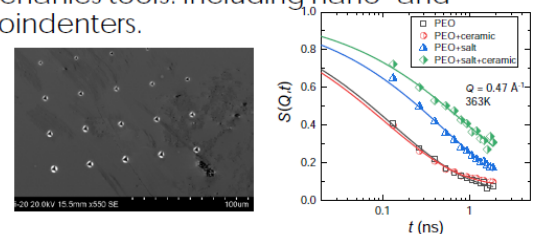

Key publications ORNL

Li distribution depends on the cathode and the duty cycle $J$ Electroceramics, 38 (2017) 222-229, DOI: 10.1007/s10832-017-0073-2.

Li-free design

J. Electrochem. Soc. 147 (2000) 517-523, DOI: 10.1149/1.1393226 Other (A-ha!)

Electrochemical stability in solid electrolytes - Schwietert et al. Nat. Mat, (2020) DOI: 10.1038/s41563-019-
$0576-0$

\section{Critical science gaps and opportunities for SSB N. Dudney}

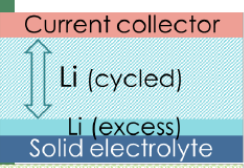

Cathode composite

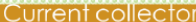

Max energy per mass \& vol dominated by active cathode
- What promotes uniform layer-by-layer plating and striping of Li metal, utilizing most of Li?

- What will improve ion transport across polymer/ceramic interfaces electrolytes

- How can we achieve and maintain full interface contact between solid materials without resorting to a liquid or soft fill layer?

- How to form robust solid-solid interfaces when there is a differential in volume changes when cycled? Strengthened by tempering?

- Can pure sintered cathode provide high areal and specific energy

- Cathode active materials that are unique to SSB, not borrowed from Li-ion.

- How can co sintering be achieved without interdiffusion. Are some interfaces stable?

- What aging processes are unique to SSB? Cycling fatigue due to accumulation of defects?

* OAK RIDGE 


\title{
Bruce Dunn (University of California, Los Angeles) \\ Solid state battery R\&D Brainstorming Session
}

\author{
Bruce Dunn \\ Materials Science and Engineering \\ UCLA
}

\section{Current Programs:}

\section{Electrochemical Materials and Devices}

Pseudocapacitive materials; ionogels, photopatternable electrolytes; solid state microdevices

\section{Materials Science Studies}

Sol-gel processing, transparent porous materials, thermoelectrics

\section{Group and Resources:}

10 Ph.D. students + postdocs; fully equipped laboratories

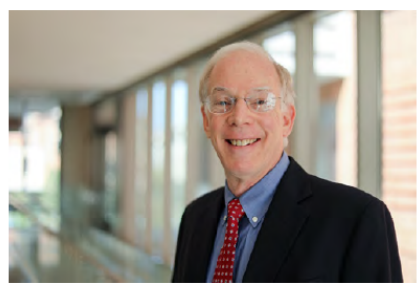
for materials synthesis and characterization

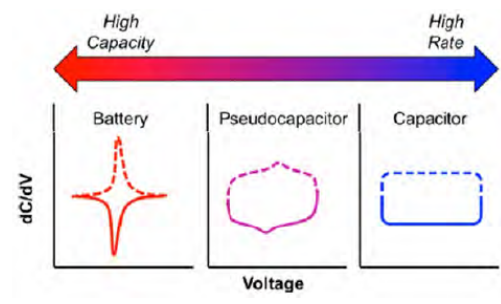

Recommended UCLA publications: D.S. Ashby, et. al. Solution-Processed lonogels ...Joule (2017) https://doi.org/10.1016/i.joule.2017.08.012. C.S. Choi et. al. Photopatternable Lithium-Ion Conducting Solid Electrolyte, Advanced Materials (2018). https://doi.org/10.1038/s41578-019-0142-z C Choi, et. al...high energy and high power pseudocapacitive materials Nature Reviews Materials, 5, 5-19 (2020) https://doi.org/10.1038/s41578-019-0142-z Some other recommended reading: J. Janek, W.G. Zeier, ..Solid future for battery development Nat. Energy. (2016) doi:10.1038/nenergy.2016.141. S. Randau, et. al. . Benchmarking All-Solid-State Lithium Batteries. Nat. Energy (2020) https://doi.org/10.1038/s41560-020-0565-1.

\section{Solid state battery R\&D Brainstorming Session Bruce Dunn}

\section{Critical Science Gaps:}

- Understand mechanisms that govern transport and properties at solid/solid electrochemical interfaces and within materials

- Determine nanoscale chemical and voltage gradients across interfaces

- Need to characterize interface regions formed by reaction at solid electrolyte/electrode interfaces

- Need to develop and validate analytical tools with emphasis on operando methods

- Processing science

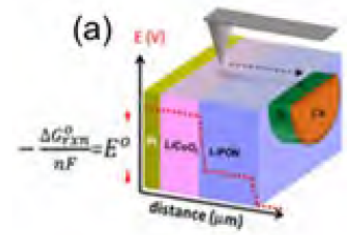

- Use of solution processing methods for fabrication and not just for synthesizing precursors

- Fast sintering methods 


\section{Stephen Harris (Lawrence Berkeley National Laboratory)}

\section{Pressure-Driven Interface Evolution in Solid State Li Metal Batteries}

- People

- Steve Harris (LBL); Jane Wang and Xin Zhang (Northwestern);

Katie Harrison and Scott Roberts (SNL)

- Unique Scope

- Multi-scale 3D time-dependent model for Li:solid electrolyte contact

- Details of the surface roughness profiles control interface resistance

- Creep under pressure

- Stress at one location and time affects contact and electrochemistry at distant locations (tens to hundreds of $\mu \mathrm{m}$ away), hours later

- We can model

- Interface impedance evolution over periods of days (Sakamoto)

- Conditions that lead to current instability

- Next steps

- Relationship between surface roughness and dendrite growth

- Effects of temperature excursions on contact and electrochemistry

Steve Harris

\section{To Reduce Gaps in Our Knowledge:}

- Study mechanical properties of real-world (not lab) Li metal

- Impurity coatings and inclusions cannot be ignored

- Do inclusions act as mechanical reinforcements?

- Are the properties of real-world Li history-dependent?

- Rethink the Monroe-Newman model for dendrite penetration

- Model designed for polymer separators in conformal contact with Li metal

- There is almost no such thing as conformal contact for Li:LLZO or Li:LGPS

- Fails to explain Li penetration through hard solid electrolytes

- Fails to explain why 5 atm inhibits dendrites in liquid electrolyte cells

- Improve relationships with industry

- Industrial partners often cultivated mainly for funding or free information

- Emphasis on technology push, rather than industry pull

- Work on 622 continued long after 811 was in production

- Focus on $\mathrm{Ah} / \mathrm{kg}$ for many years, at the expense of $\mathrm{Wh} / \mathrm{l}$, of greater importance to the auto industry 


\section{Erik Herbert (Michigan Technological University)}

\section{INTRODUCTION \& SSB RESEARCH ACTIVITIES}

\section{OUR COLLABORATIVE TEAM}

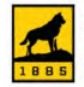

Michigan

Technological

University.

- Erik Herbert

- Steve Hackney

- Nancy Dudney

\section{SCOPE OF OUR PROGRAM}

- Coupling between structure-property relationships \& device performance

- Metallic lithium \& all classes of SSEs

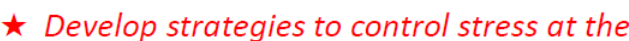
buried Li/SSE interface during charging

\section{UNIQUE CAPABILITIES, KNOWLEDGE \& RESOURCES}

- Small-scale mechanical characterization

- 1D \& 2D systems, dedicated glove box

- Control state of stress, strain \& strain rate

- Post \& in-situ cycling

- In-situ SEM

$-10 \leq T \leq 80^{\circ} \mathrm{C}$

- Analytical modeling

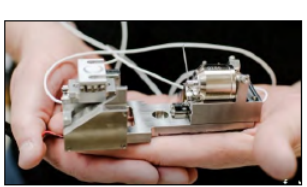

RECOMMENDED READING FROM OUR PROGRAM

"On the mechanisms of stress relaxation and intensification at the lithium/solid-state electrolyte interface," J. Mater. Res. 34 21, 3593-3616 (2019).
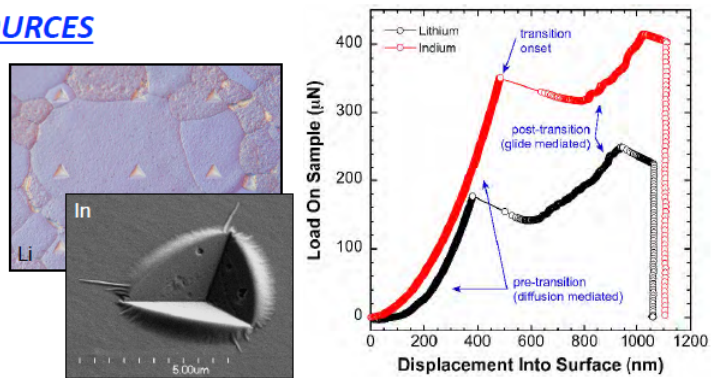

\section{RECOMMENDED READING FROM OTHER PROGRAMS}

L. Barroso-Luque, Q. Tu \& G. Ceder, "An Analysis of Solid-State Electrodeposition-Induced Metal Plastic Flow and Predictions of Stress States in Solid Ionic Conductor Defects," J. Electrochem. Soc. 1672 (2020).

\section{CRITICAL SCIENCE GAPS \& OPPORTUNITIES FOR SSBS}

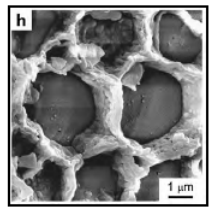

Intergranular propagation of $\mathrm{Li}$ through LLZO grain boundaries

Cheng et al., Electrochimica Acta 223 (2017)

\section{In the Absence of Stress-Free Lithium Plating}

1. What are the stress relaxation mechanisms in Li that control stress at the Li/SSE interface during charging?

- $1 \leq n \leq 6+$ and $0.5 \leq \sigma_{y} \leq 100 \mathrm{MPa}$

- How do these mechanisms depend on: (1) Defect Geometry; (2) Length Scale; (3) Current Density (strain rate); (4) Temperature; (5) Cycling Conditions; and (6) Stack Pressure

- How do these mechanisms evolve with time? (Transient, Transition \& Steady-State behavior)

2. How does the SSE contribute to stress relief?

- Creep \& reversible flow in amorphous SSEs observed. Mechanisms? Merits further investigation.

- Volume vs. non-volume conserving deformation. How are these mechanisms affected by chemistry and/or composition?

- How do we quantify the conditions \& criteria for crack nucleation vs. crack propagation $\left(K_{l c}\right)$ ?

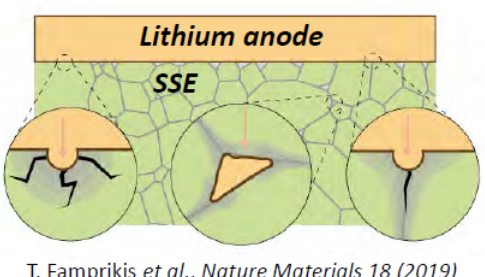

T. Famprikis et al., Nature Materials 18 (2019)

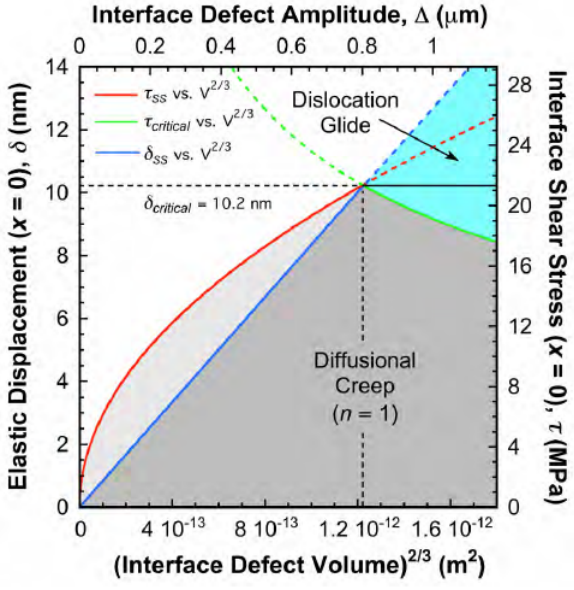




\section{Subra Herle (Applied Materials) \\ ORNL Solid State Battery}

Personal introduction:

- Subramanya (Subra) Herle Ph.D. Director, Distinguished Member of Technical Staff, Office of the CTO, Applied Materials

- CTO of energy storage, joined Applied Materials in 2010

- Worked for Panasonic battery division over 6 years

- Ph.D. in solid state chem, IISc Bangalore; worked in Ames lab; Univ. Tubingen (Humboldt fellowship) and Univ Waterloo, Canada

How my activities important to SSB research?

- Scope of my programs/researchers

- New market: Identify opportunities to develop technologies for the growing energy storage market

- Technology: Identify high TRL level programs to accelerate HVM equipment development for energy storage market

- Validation: Collaborate with start-up/National Lab/Univ. to get proof point to de-risk technical component of the business

- What is unique to my capabilities, knowledge, resources

- Core Capability: We have S2S and R2R deposition technologies and tools in the dry room

- Infrastructure: Electrochemical test capabilities, Metrology (in-line and off line) for film thickness measurement

- Global Team: Team of electrochemical technologists, process engineers, hardware and software engineers

- What I recommend you read from my program

- Pre-lithiation/Li anode: Limited info at the 2019 Florida International Battery Seminar invited speaker

- What I recommend you read from literature,

SSB Review: Simon Randau, Nature Energy vol 5 (2020) 259-270 Benchmarking the performance of all-solid-state lithium batteries

SSB Challenges: Paul Albertus et. al. Nature Energy vol 3 (2018) 16-21 Status and challenges in enabling the lithium metal electrode for high-energy and low-cost rechargeable batteries

All SSB Ah cell proof point: Yong-Gun Lee et. al Nature Energy vol 5 (2020) 299-308 High-energy long-cycling all-solid-state lithium metal batteries enabled by silver-carbon composite anodes

- Interface (NMR): Chuang Yu et. al, Nature Commun. vol 8 (2017) 1086 Accessing the bottleneck in all-solid state batteries, lithium-ion transport over the solid-electrolyte electrode interface

- Electromechainal instability and interface: Tushar Swami Ph.D. thesis MIT 2018 https://dspace.mit.edu/handle/1721.1/118732 Electro-chemo-mechanical instabilities at interfaces in all-solid-

- Benchmarking (Li-ion): Jessie E. Harlow Journal of The Electrochemical Society, 166_13 (2019) A3031-A3044 A Wide Range of Testing Results on an Excellent Lithium-lon Cell Chemistry to be used as Benchmarks for New Battery Technologies

- Li-ion cost Learning curve (Li-ion): I-Yun Lisa Hsieha et. la. Applied Energy 239 (2019) 218-224. Learning only buys you so much: Practical limits on battery price reduction

2. | Applied Materials Confidentia

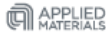

\section{Critical Science Gaps and Opportunities for SSBs}

Objective: Develop "Ah class" solid state electrochemical secondary cell with equivalent or superior cell performance vs Li-ion and low cost (\$/kWh).

Mission Statement: ASSB, SSB, Hybrid SSB, SSB with polymer/gel electrolyte $\rightarrow$ Performance based vs material physical property $\rightarrow$ helps to focus efforts

Playbook: Think big, test small, fail fast and learn quick $\rightarrow$ R\&D vs C\&F; Data $\rightarrow$ Boundary conditions?

Collaboration: Leverage key expertise across organization/institutions and leverage infrastructure $\rightarrow$ accelerate development cycle

Gaps, challenge and research considerations:

\begin{tabular}{|l|l|l|}
\hline Topics & Context $\rightarrow$ Perspective & Paterials \\
science & $\begin{array}{l}\text { If solved would open new opportunities for materials } \\
\text { and interface that will impact development of SSBs: } \\
\text { Materials that could be potentially fabricated into rolls } \\
\text { using existing manufacturing techniques. } \\
\text { Less air sensitive materials with superior mechanical } \\
\text { and performance benefits }\end{array}$ \\
\hline Processing & $\begin{array}{l}\text { New research can lead to innovative processing of } \\
\text { materials to achieve materials or interface control and } \\
\text { properties that are unique from conventional } \\
\text { processing } \\
\text { Low temperature deposition of few } \mu \text { m thick material } \\
\text { (eg. pin hole free for electrolyte) and post processing } \\
\text { reduce thermal impact on the substrates. }\end{array}$ \\
\hline Battery & $\begin{array}{l}\text { New designs would take advantage of unique material } \\
\text { processes and mechanisms. (This is not a battery } \\
\text { optimization through design.) } \\
\text { Define prototype Ah capacity SSB cell design for min } \\
\text { qualification }\end{array}$ & . \\
\hline architecture
\end{tabular}

Gaps

Provide template to researchers to capture all details $\rightarrow$ Accurately record material handling conditions

de SSB community

Thickness, tap density, density, surface roughness,

processing ambient \& control, standardized procedure to

report data, voltage window \& cyclic voltammetry, Over

potential vs current density plots

Voltage stability discrepancy between experimental and theoretical

groups

Interface studies with Li metal and measurement techniques.

Identify optimal material and thickness including interface layers Streamline process flow paths $\rightarrow$ build device architecture from the 'substrate'.

Develop unique test kits to analyze and understand interface and analytically quantify inventory counting of active vs inactive components, lost active components per cycle and calendering conditions etc.

Prototype Ah cell: Clearly identify the parameters and performance success criteria

olar electrode for SSB and practical anufacturing considerations

\section{Comments}

Archive quality data in a single weblink for researches to access

Provide computational tools for researchers that will force fundamental material properties as input in standard format Clearly identify TRL's; Have periodic loop reviews Material company partner $\rightarrow$ Material de-risking at early TR

Train researchers software to get high level trends for process conditions (JMP) Clearly identify TRL's

Have periodic loop reviews $\rightarrow$ Equipment company partner

Clearly identify TRL's

Develop extended $\mathrm{BatPaC}$ or equivalent model for SSB

Loop review $\rightarrow$ Batter company partner 


\section{Joseph Libera (Argonne National Laboratory) AEROSOL SYNTHESIS FACILITIES FOR LLZO Joseph Libera (Argonne national Laboratory)}
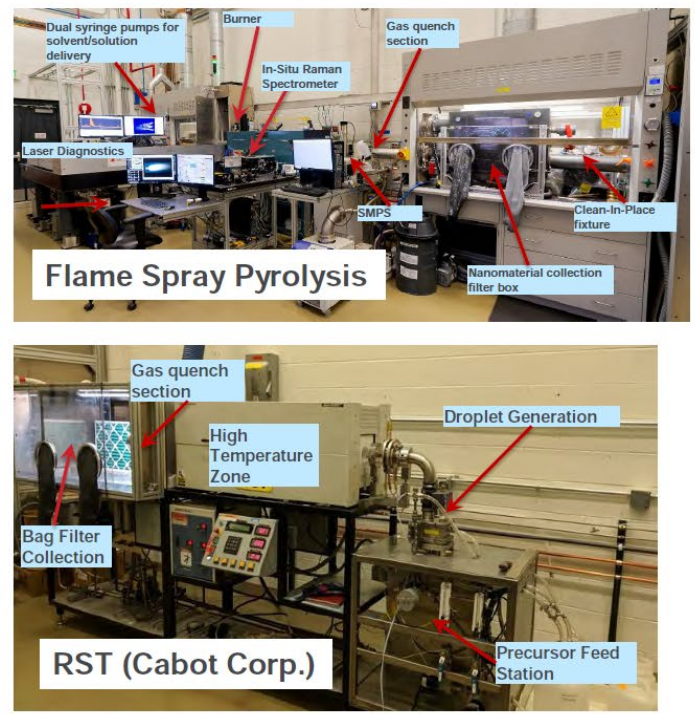
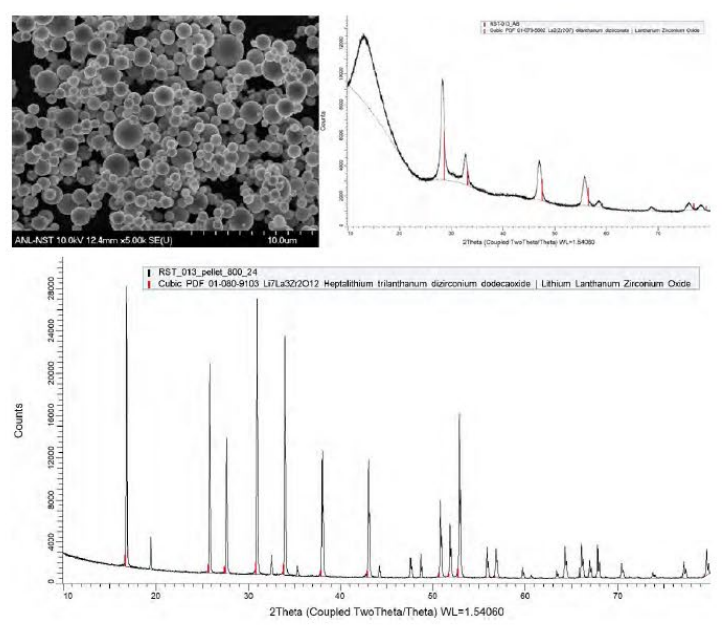

(2) ENERGY Y

Argonne $\mathbf{A}$

Joseph Libera

\section{AEROSOL SYNTHESIS FACILITIES FOR LLZO}

- Cost effective strategies for commercial Scale Production of Garnet SSE's.

Development of commercial scale SSB processes must utilize sensible commercial scale feedstock's

- FSP: Flame spray pyrolysis

- combustion process using organic solvent solutions of organic or inorganic metal salts

- Produces nano-scale powders

- RST: Reactive Spray Technology (Cabot) no-flame process

- Non-combustion process, Aerosol's are generated and passed through a high temperature zone.

- Produces micron-scale powders

- FSP and RST LLZO powders

- As-produced are LZO + Li salts.

- Calcination directly to cubic LLZO occurs at $700^{\circ} \mathrm{C} . \mathrm{CO}_{2}$ and/or water are liberated during the calcination.

- Manufacturing schemes for SSB taking advantage of the low-temperature LLZO is suggested.

- Co-sintering of LLZO and cathode enabled by $<800^{\circ} \mathrm{C}$ sintering.

- Nano/micro-scale green powder blends for optimizing mixing properties with cathode phases.

- Need to manage carbonate/hydroxide during manufacture. 


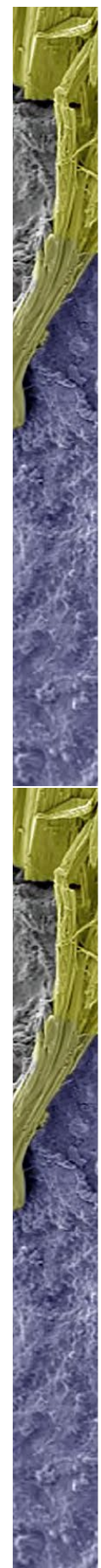

\section{Dongping Lu (Pacific Northwest National Laboratory)}

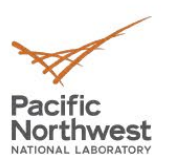

\section{Development of High Energy Li-S Battery Supported by VTO/BMR}

Dongping Lu/PNNL

Project focus:

- Project efforts are aimed at key barriers of Li-S batteries: low practical system energy, low S utilization rate at high-loading, and limited cycling life at lean electrolyte conditions.

- Fundamental understanding of key factors (material \& electrode levels) that affect electrode preparation, S reaction kinetics, and cell lifespan at realistic high S loading and lean electrolyte conditions.

- Optimize material/electrode structures to realize a high $\mathrm{S}$ utilization rate in low-porosity $\mathrm{S}$ cathodes to boost both cell specific energy $(\mathrm{Wh} / \mathrm{kg})$ and energy density $(\mathrm{Wh} / \mathrm{L})\left(>4 \mathrm{mg} \mathrm{S} / \mathrm{cm}^{2},<50 \%\right.$ porosity)

- Tackle the challenges of low ionic-conductivity of $\mathrm{S} / \mathrm{Li}_{2} \mathrm{~S}$ by utilizing solid electrolytes (SE), which requires high-quality and compatible solid electrolytes.

Pacific Preliminary efforts in addressing SE materials challenges Northwest

- Challenges of SE particle control

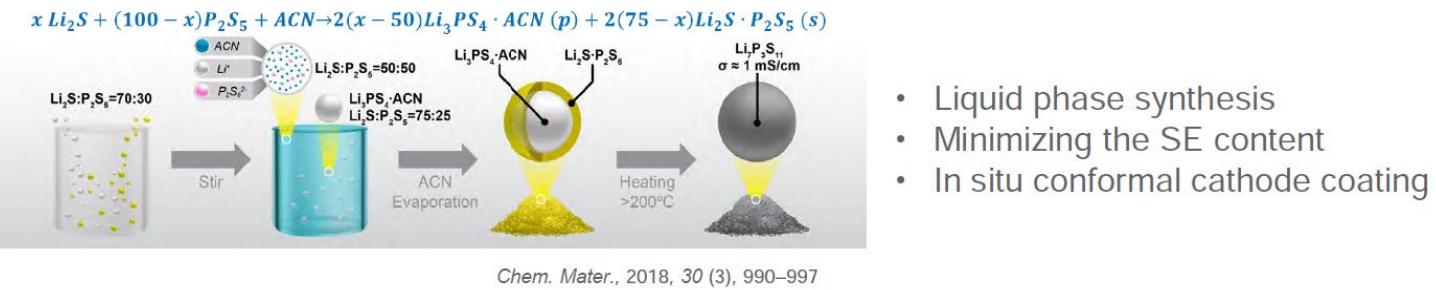

- Challenges of Li/SE interfacial stability
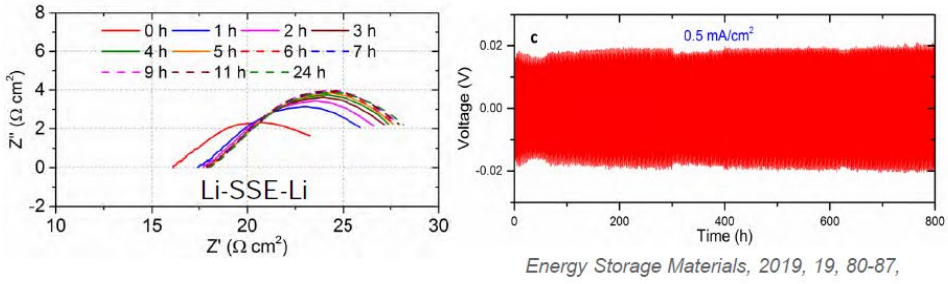

$\mathrm{Li}_{7} \mathrm{P}_{2} \mathrm{~S}_{8} \mathrm{X}(\mathrm{Cl}, \mathrm{Br}, \mathrm{I}, \ldots .$.

- Low and stable Li/SE interface $\left(<5 \Omega \mathrm{cm}^{2}\right)$

- Long-term cycling at high current 


\section{Steve Martin (Iowa State University)}

Steve W. Martin - Iowa State University - Development of Thin Robust High $\mathrm{Li}^{+}$Ion Conductivity Glassy Solid Electrolytes

- Glasses can be compositionally tailored to meet a wide range of performance needs

- Glasses can be processed in large volume and drawn into
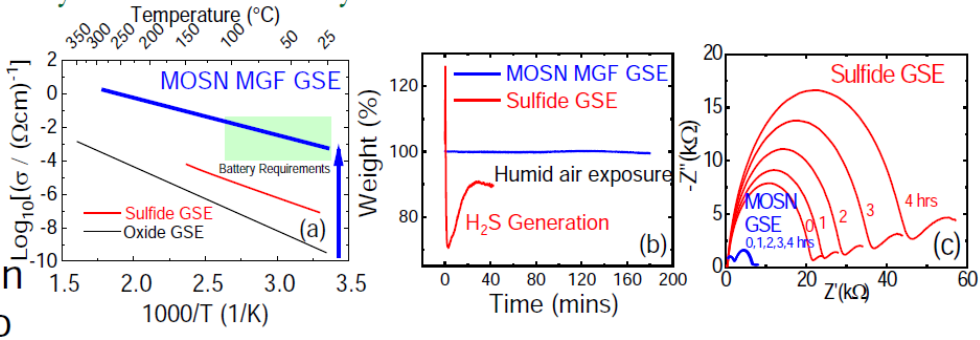
continuous long thin films at low cost

- Glasses can exhibit optimized solid electrolyte properties in a format well suited to all solid state batteries
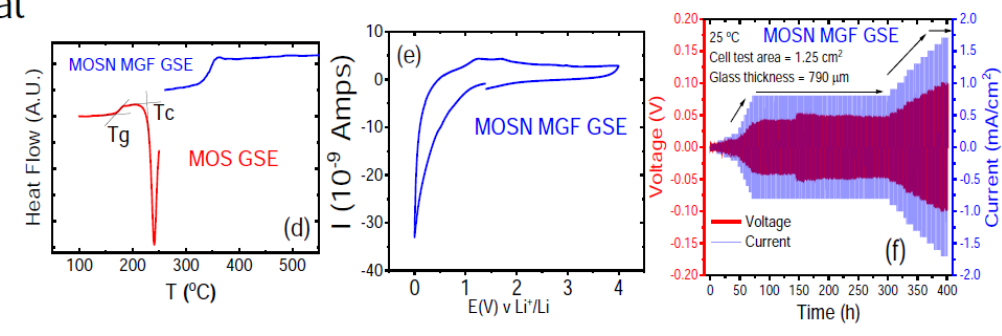

swmartin@iastatge.edı

Iowa State University of Science \& technology

Steve W. Martin

Critical Science Gaps and Opportunities - Lithium/Sodium Anodes

- What is the role of homogeneous atomically smooth and uniform surfaces?

- What happens to Li/Na plating/stripping in the absence of surface defects, composition gradients, grain boundaries, etc.?

- Can SE Chemistry be created to produce a truly thermodynamic stable interface instead of relying on kinetic stability and/or a stable SEI?

- Can mixtures of anions and cations produce a thermodynamically stable interface, yet enable critical SE properties such as conductivity, resistance to dendrites...

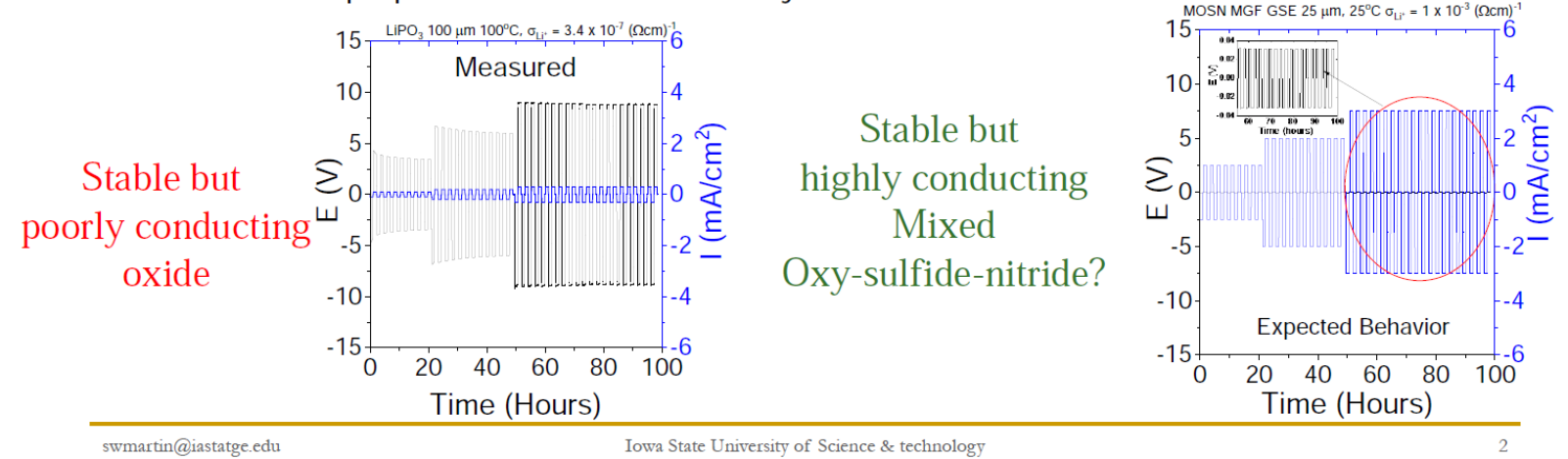




\section{Bryan McCloskey (University of California, Berkeley) \\ McCloskey Lab: nonaqueous polyelectrolyte solutions}

McCloskey

- Can we develop 'soft' electrolytes with unique transport properties (high transference number and conductivity, low interfacial impedance)?

- Synthesize single ion-conducting polymers.

- Prepare polyelectrolyte solutions to study influence of solvent composition, polymer concentration, molecular weight.

- Characterize transport properties using a combination of AC impedance, pulsed field gradient NMR (self-diffusion coefficients), viscosity, electrochemical methods.

- Use molecular dynamics to understand molecular underpinnings of ion transport trends.

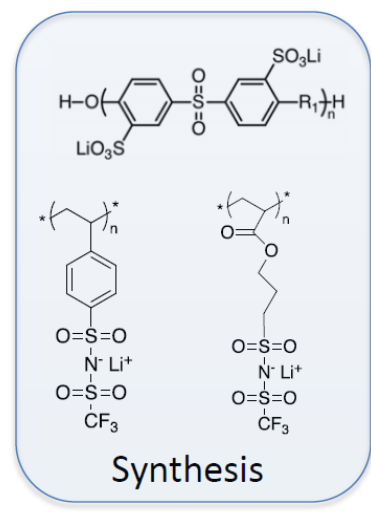

Berkeley

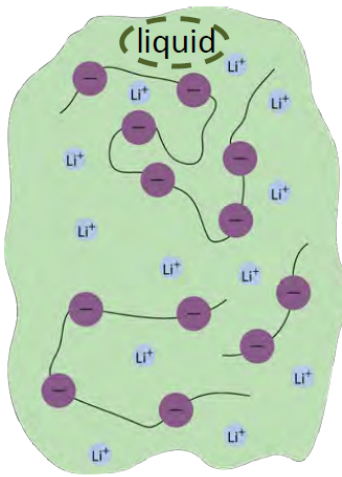

Preparation

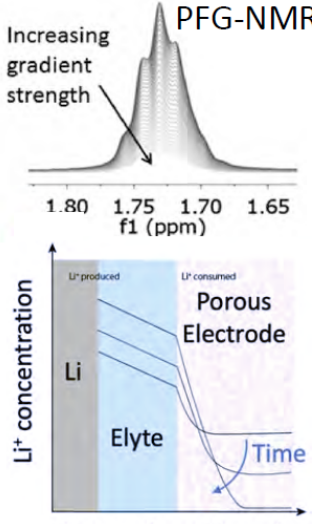

Distance from Li anode

Characterization
Solvent-Separated Ion Pair (SSIP)

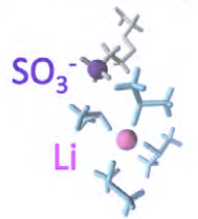

Charged
Lennard-Jones $\rightarrow$ Bead-spring chain Lennard-Jon particles

†

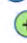

+ Hove Explicit solvent (neutral $†$ Collaboration, K. Persson lead

Research gaps for polymers

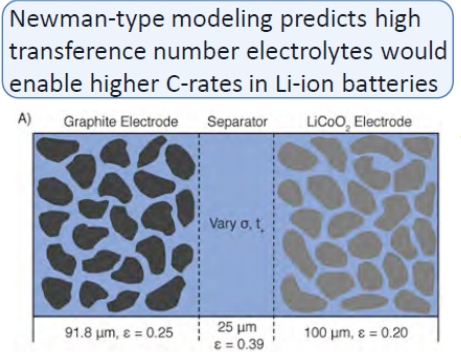

C)

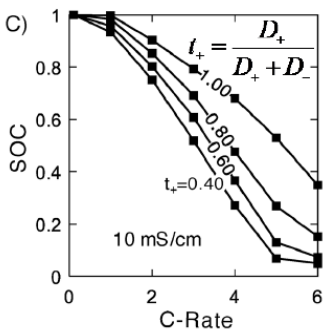

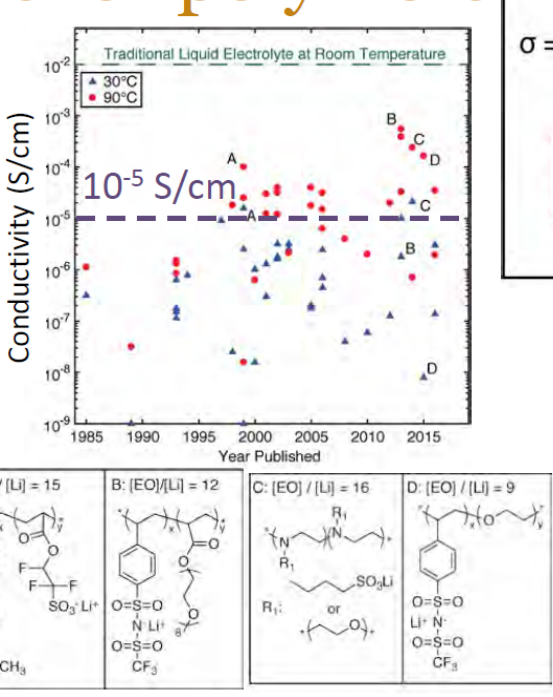

Dry polymer electrolytes suffer from low conductivity (each point is a unique polymer)

$$
\sigma=A \exp \left(-\frac{E_{a}}{R\left(T-T_{0}\right)}\right)
$$

For polymers:

$\downarrow E_{a} \Rightarrow \downarrow A$
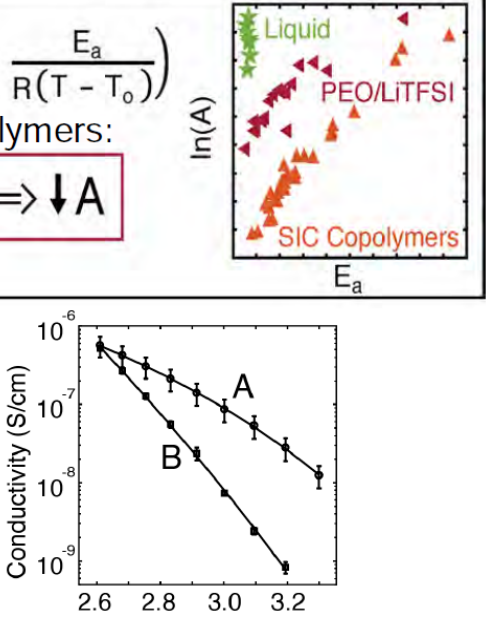

Temperature $(1000 / \mathrm{K})$

The 'compensation effect' indicates a tradeoff between segmental motion and ion solvation Our articles of interest:

1. ACS Energy Letters (2017), 2(11), 2563.

2. J. Phys. Chem. Lett. (2015), 6(22), 4581.

3. Macromolecules (2017), 50(10), 3831. 


\section{Matthew McDowell (Georgia Institute of Technology)}

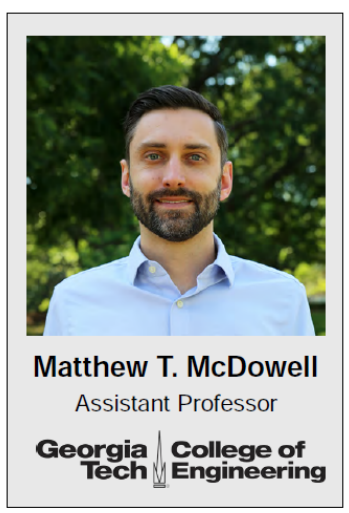

Key Recent Literature:

1. R. Koerver, ..., W. G Zeier, J. Janek. Chemo-mechanical expansion of lithium electrode materials - on the route to mechanically optimized all-solid-state batteries. Energy Environ. Science (2018) 11, 2142-2158.

2. Kasemchainan, J. et al. Critical stripping current leads to dendrite formation on plating in 3 lithium anode solid electrolyte cells. Nature Materials (2019) 18, 1105-1111.

3. Wang, M. J., Choudhury, R. \& Sakamoto, J. Characterizing the Li-Solid-Electrolyte Interface Dynamics as a Function of Stack Pressure and Current Density. Joule (2019) 3, 9, 2165-2178.

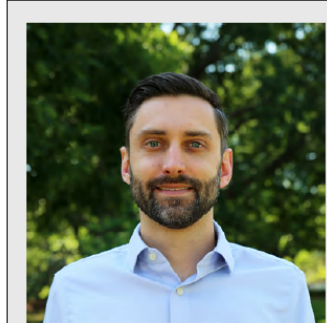

Matthew T. McDowell Assistant Professor

Georgia College of Tech Engineering

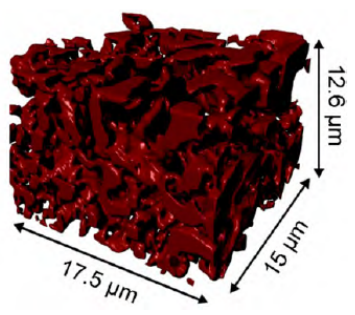

S. Han, ..., McDowell, Chem. Mater. (2020), 32 (6), 2461-2469.
My Solid-State Efforts:

- In situloperando characterization of interface and electrode evolution ( $\mathrm{x}$-ray, TEM)

- Chemo-mechanics in solid-state batteries

- Interfacial engineering

- $\sim 4$ researchers in my group

Unique capabilities/knowledge:

- Bridging length scales with in situ/operando

- Linking electrochemistry to solid mechanics

\section{Relevant Group Publications:}

1. J. Lewis, ..., McDowell, ACS Energy Lett., (2019), 4, 2, 591-599.

2. J. Tippens, .... McDowell, ACS Energy Lett., (2019), 4, 6, 1475-1483. 3. J. Lewis, ..., McDowell. Trends in Chemistry (2019), 1, 9, 845-847.

4. F. Cortes, ..., McDowell. J. Electrochem. Soc. (2019) 167, 050502.

More forthcoming!

\section{Critical Science Gaps:}
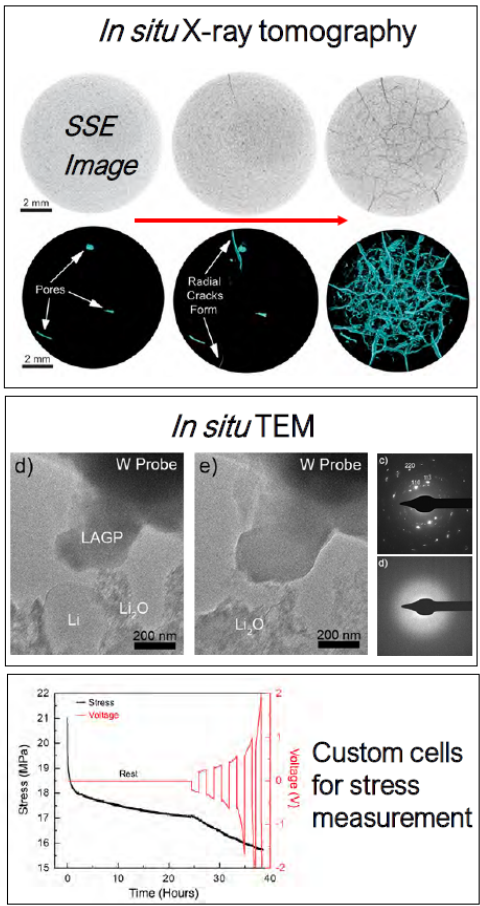

Matthew T. McDowell

1. Need to better understand how alkali metals ( $\mathrm{Li}$ and $\mathrm{Na}$ ) evolve during plating/stripping: morphology changes, filament growth, unstable interfaces.

2. Understand the influence of alloying metals on the behavior and dynamic evolution of lithium metal anodes.

See recent papers:

1. Y.-G. Lee, et al. Nature Energy, (2020) 5, 299-308.

2. T. Krauskopf, et al. Adv. Energy Mater. (2019) 9, 44, 1902568.

3. Determine how interface instabilities and metal filament growth are interrelated.

4. Control structural evolution of composite cathodes across scales. 


\title{
Jagjit Nanda (Oak Ridge National Laboratory)
}

\author{
Jagjit Nanda \& Team, Oak Ridge National Laboratory
}

Ongoing R\&D Effort in Solid Electrolytes, Membranes and Interfaces

- Sulfide based superionic solid electrolytes \& processing

- Enabling solid electrolyte cathode interfaces - Integrated Approach

- Mechanically robust polymer composites membranes and electrolytes for Redox flow and lithium batteries

- Novel solid electrolytes

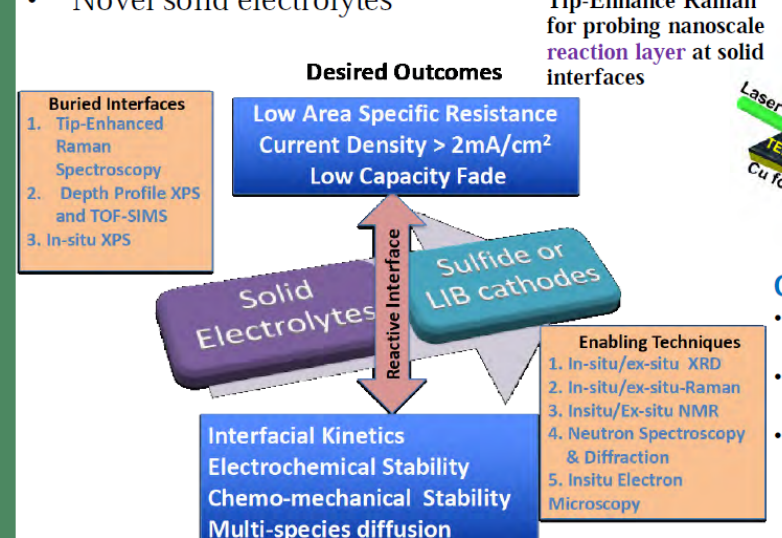

Multi-species diffusion

* OAK RIDGE
Key Technical Barriers

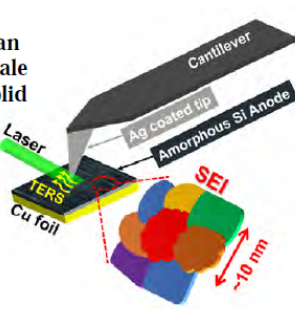

Current Focus:
Ethan Self Guang Yang Yan-Wu Tsai

- Synthesize superionic sulfide SEs using low temperature scalable, solvent-mediated routes

Develop sulfide/polymer composite SEs which can be tape cast into layers $<30 \mu \mathrm{m}$ thick.

Improve cathode/sulfide SE compatibility via interfacial buffer layers/coatings.

Relevant References from group and others

MRS BULLETIN,VOL 43. OCT. 2018 ACS Energy Lett. 2020, 5, 910-915

Joule 3, 1-19, August 21, 2019 ACS Appl. Mater. Interfaces 2019, 11, 42042-42048

J. Mater. Chem. A, 2017, 5, 22750-22760 ACS Appl. Mater. Interfaces 2019, 11, 42186-42196

\section{Critical Science Challenges and Gaps}

Jagjit Nanda

Develop further understanding on factors that determines uniform Li-plating and stripping

for any model solid electrolyte

- Surface defects, inhomogeneity, morphology, texture of current collectors and thin Li-foils

Fabricating thin ( 30 microns) mechanically robust, dense (and preferably flexible) solid electrolyte

separators - How much we have succeeded?

What is the current state-of-art?

Have we made enough progress? Moisture and chemical stability for sulfides SEs.

Do we understand key factors that determine wettability between solid-solid interfaces and its link to ion-

transport?

- Local microstructure that determines the contact area between solid electrolyte and electrode surface

- External pressure to improve wettability depends on the nature of SE and electrode materials

Hard-Hard; Hard-Soft and Soft-Soft

- Very high stack pressure for cycling solid state batteries may not be practical in long term

- Computation modelling, AI and ML techniques can help or aid deciding compatible material systems interfaces etc.

Mechanical Constriction as an approach to provide internal stress for example by creating core-shell geometry as an alternative to high stack pressure. Strain Engineering to improve electrochemical stability

NATURE COMMUNICATIONS | (2018)9:4037; Small 2019, 1901470

Variability in performance data both at materials and cell level for SSB's

Wide variation of ionic conductivity values for the same solid electrolyte system from different groups

Unoptimized cell design, non-standard experimental methods, no baseline metrics

OAK RIDGE

Interesting study - ACS Energy Lett. 2020, 5, 910-915 


\section{Jeff Sakamoto (University of Michigan)}

\section{M}

Jeff Sakamoto

University of Michigan

Mechanical Engineering \& Materials Science and Engineering

Processing, Electrical \& Mech Props

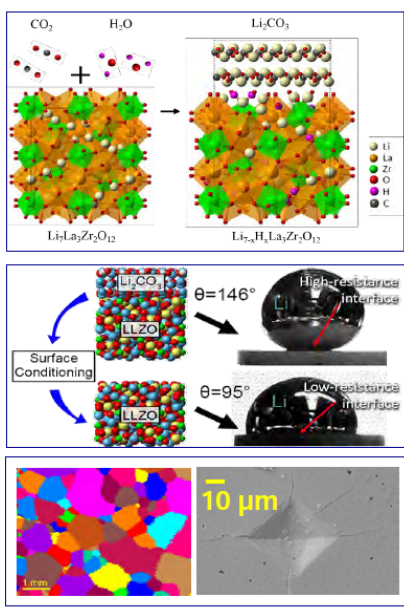

Mechano-Electrochemical Coupling
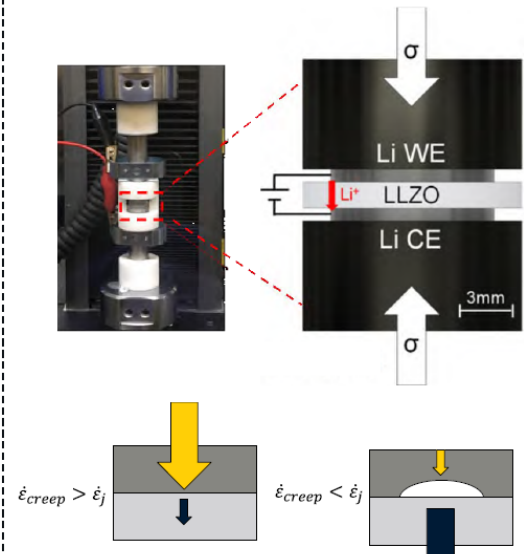

M. J. Wang et al., Joule, (2019).
Does a Material Property Control CCD?

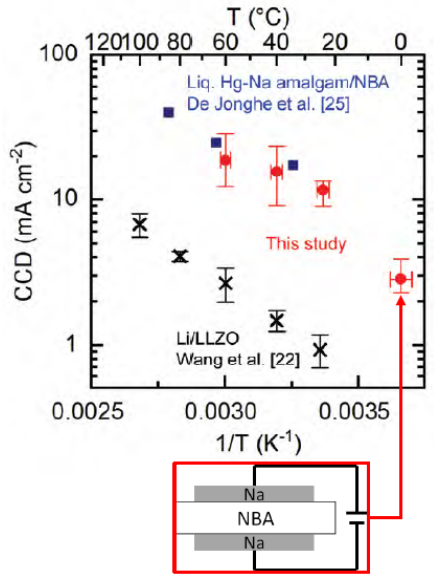

M.-C. Bay, M. J. Wang, et al., Adv. Energy Mater., (2020).

\section{Questions and Unmet Needs}

Jeff Sakamoto

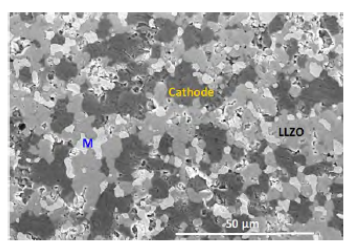

Ceramic Composite Cathode

- Mitigating inter diffusion \& stresses during densification is possible.

- However, cycling behavior is not well-understood.

- Does physical confinement affect cycling behavior?

- Are the interface kinetics sufficient to allow cycling?

- Unmet need: Techniques to analyze stresses and chemistry at buried interfaces.

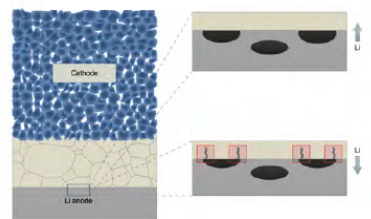

Chemical \& Physical Phenomena at the Alkali Metal-Solid Electrolyte Interface

- Does a material property affect $C C D^{\text {stripping }} \& C C D^{\text {plating? }}$

- How is flux balance maintained at interfaces?

- Is wetting maintained during cycling and if not why not?

- Unmet need: Techniques to analyze buried interfaces in operando that have adequate special and temporal resolution.

\section{Cathode/Catholyte interface stability and kinetics}

- Hybrid electrolyte schemes are attractive due to ease of fabrication.

- However, little is known about inorganic/organic electrolyte interfaces.

- How does solvation/de-solvation work and is there and SEI?

- Unmet need: Need to understand chemical-electrochemical phenomena. 


\section{Sanja Tepavcevic (Argonne National Laboratory)}

Argonne

"Surface Science" Approach Tepavcevic

Deposition Methods

Combination of physical and chemical methods for deposition of metallic $\mathrm{Li}$ and oxide thin films:

$\checkmark$ Sputtering

$\checkmark$ E-beam Deposition

$\checkmark$ Electrochemical

$\checkmark$ Pulsed Laser Deposition

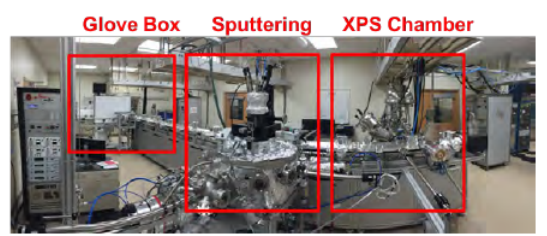

Characterization Methods

Various ex situ and in situ experimental tools and first principles modeling:

$\checkmark$ X-Ray Photoelectron Spectroscopy

$\checkmark$ Impedance

$\checkmark$ DFT and Molecular Dynamics

$\checkmark$ Soft X-Ray Spectroscopy

$\checkmark$ Synchrotron XRD, HANES

$\checkmark$ Fourier Transform Infrared Spectroscopy

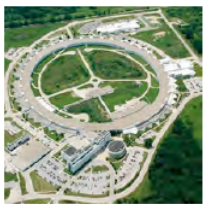

> Scope: Develop and use state of the art experimental and computational characterization techniques to establish functional links between stability, selectivity, transport and conductivity of electrochemical interfaces and bulk materials in Li-ion battery systems.

$>$ Strategy: Fast transfer of fundamental knowledge from model to real world systems.

> Publication: Dopant-Dependent Stability of Garnet Solid Electrolyte Interfaces with Lithium Metal Zhu, Y., Connell, J. G., Tepavcevic, S., Zapol, P., Garcia-Mendez, R., Taylor, N. J., Sakamoto, J., Ingram, B. J.,Curtiss, L. A., Freeland, J. W., Fong, D. D., Markovic, N. M., Adv. Energy Mater. 2019, 9, 1803440.

> A-ha: Dissolution of Lithium Metal in Poly(ethylene oxide) Michael D. Galluzzo, David M. Halat, Whitney S. Loo, Scott A. Mullin, Jeffrey A. Reimer, and Nitash P. Balsara ACS Energy Letters 20194 (4), 903-907
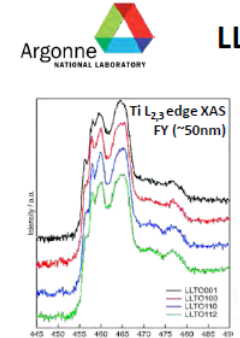

LLTO/Li Interface Study
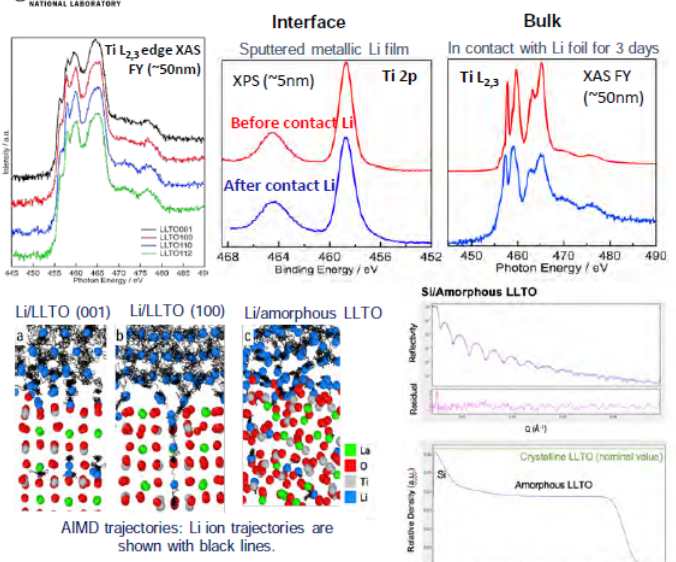

Conclusions:

- Long-term bulk reactivity of crystalline LLTO with abundant Li source is orientation-independent.

- Amorphous LLTO film found to be stable in contact with $L i$

- AIMD simulations suggest that reactivity of LLTO films towards $L i$ metal correlates with feasibility of Li ion exchange in LLTO.

- Amorphous LLTO films are significantly less dense ( $70 \%$ nominal density) but highly uniform throughout their thickness with no surface layer formation

Questions:

- Amorphous LLTO low reactivity due to low conductivity?

- Mechanistic understanding of differences in reactivity between amorphous and crystalline LLTO electrolyte with Li

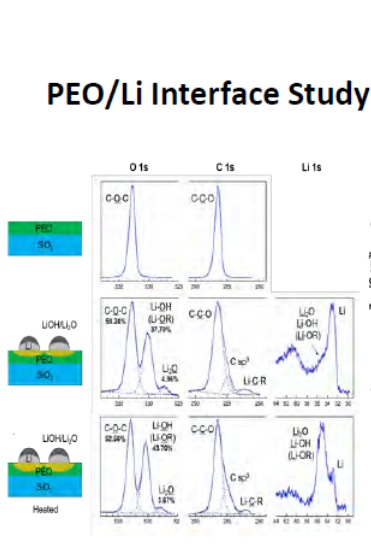

Tepavcevic
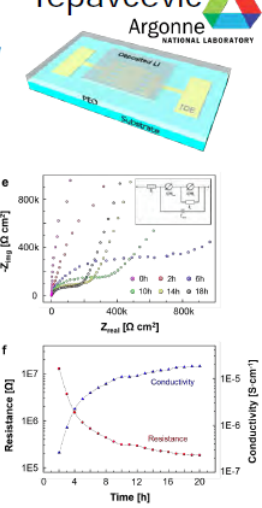

Conclusions:

- PEO's ether bonds reacts with Li metal

- Conductivity increases two orders of magnitude with 400nm thick Li film deposited on 150nm thick PEO

- EIS (Au electrodes) confirms change in bulk conductivity

- DFT modeling of reactions at a Li/PEO Interface finds Li reaction with $\mathrm{C}$-O the most probable

- Resulting $\mathrm{Li}-\mathrm{O}$ bond is ionic $\left(\mathrm{Li}^{+}-\mathrm{O}^{-}\right)$and can account for the observed ionic conductivity

Questions:

- In-situ salt formation turns PEO into single Li-ion conductor?

- Does presence of large, immobilized anion groups enable dominant contribution of $\mathrm{Li}^{+}$ions to the ionic conductivity?

Mechanism of Li-ion transport 


\section{Eric Wachsman (University of Maryland) \\ Eric Wachsman, University of Maryland - Solid State Battery Research}

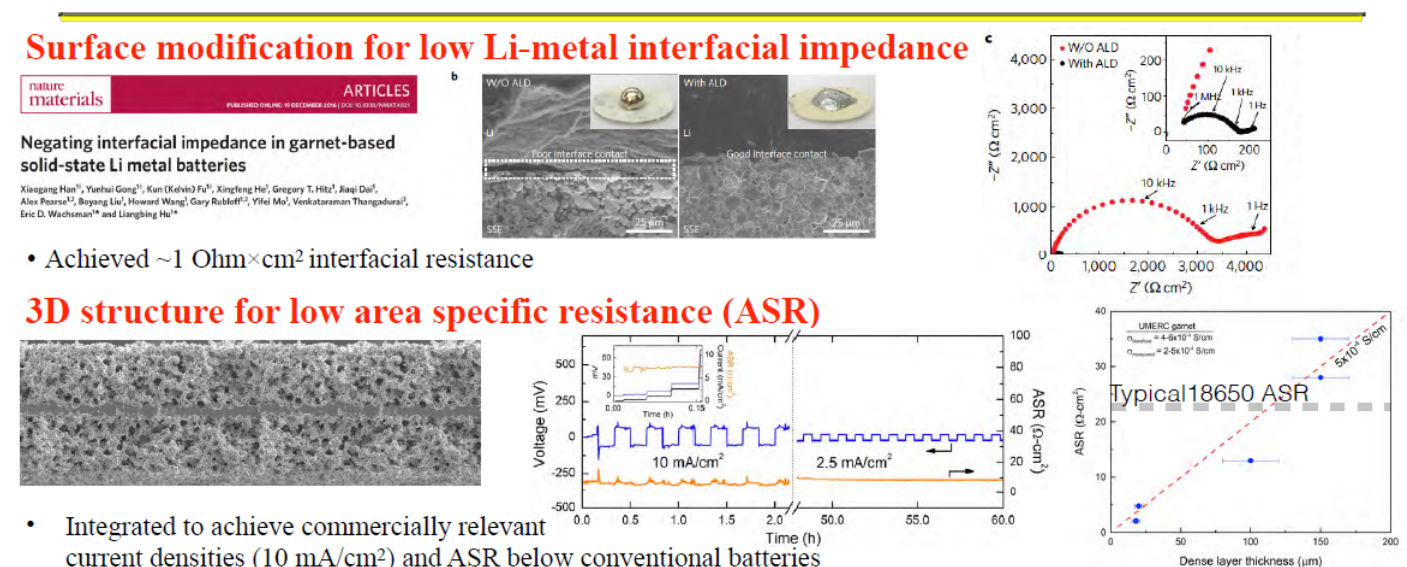

Integrating for all solid-state batteries
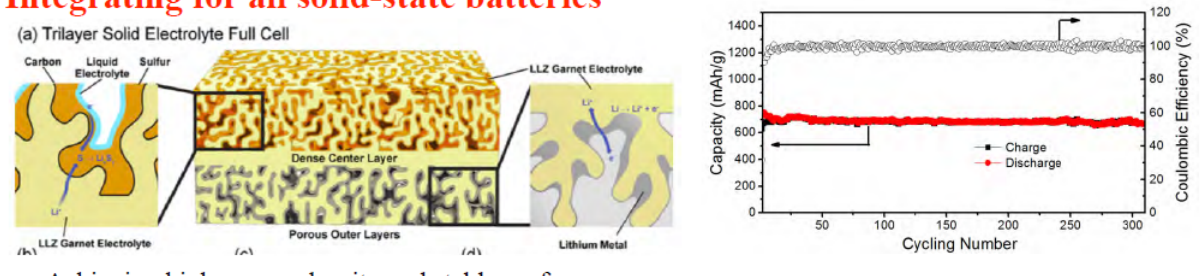

- Achieving high energy density and stable performance

8. MARYLAND ENERGY

\section{Eric Wachsman, University of Maryland - Critical Science Gaps}

I'm prepared to talk about:

- Cathode interfacial impedance

- Due to processing

- During cycling

- Electro-chemo-mechanical expansion

- Differences between cathode and electrolyte

Also need to be addressed:

- Fundamental understanding of solid-state degradation mechanisms

- SSEI formation?

- Li -metal migration during cycling

- Electrical contact and stack pressure

- Manufacturing techniques, automation, and scale-up

9. MARYLAND ENERGY 


\section{Chunsheng Wang (University of Maryland)}

\section{Dr. Chunsheng Wang at UMD Research Capability}

Materials Science:

> Current collector/Li anode/SSE interface: Lithiophlic substrate (Li-M alloy) and lithiophobic interface/interphase (LiF-rich) at Li/solid state electrolyte (SSE) to (1) promote layer by layer Li plating/striping; (2) meet expectation for high density SSE; (3) suppress Li dendrite nucleation.

Publication: Sci. Adv., 2018, 4 (12), eaau9245; Nat. Energy, 2019, 4 (3), 187-196.

> SSE thermodynamic and kinetics calculation: Capable of multi-scale computations including angstroms, nanometers, micrometers and millimeters scales using first-principles calculations based density function theory and molecular dynamics at nanometers (Gaussian, VASP, and LAMMPS packages) and phase-field and continuum simulation. Publication: Sci. Adv., 2018, 4 (12), eaau9245.

> Organic/polymer cathodes: A organic 4-(phenylazo) benzoic acid lithium salt (PBALS) cathode with strong bonding to LPS solid electrolyte via the ionic bonding for long cycle life. Publication: Angew. Chem. Int. Ed., 2018, 57, 8567-8571.

Processing science:

> A solution casting approach to prepare the mixed-conductive $\mathrm{Li}_{2} \mathrm{~S}$ cathode

$>$ Melting-casting strategy to fabricate $\mathrm{Na}_{2} \mathrm{~S}-\mathrm{Na}_{3} \mathrm{PS}_{4}-\mathrm{CMK}-3$ nanocomposites

$>\mathrm{Li}_{2.3-\mathrm{x}} \mathrm{C}_{0.7+\mathrm{x}} \mathrm{B}_{0.3-\mathrm{x}} \mathrm{O}_{3}$ solid electrolyte interphase enabled all ceramic lithium battery Publication: Nano Lett., 2016,16, 4521; ACS Nano, 2018, 12 (4), 3360-336; Joule, 2 (2018) 497

Battery Architecture:

> 3D Kevlar nonwoven supported thin ( 100um) LPS electrolyte and 3D metal form supported high-loading $\mathrm{Li}_{2} \mathrm{~S}$ cathodes $\left(\sim 7 \mathrm{mAh} \mathrm{cm}^{-2}\right)$ enabling $1^{\text {st }}$ cycle energy density of $370 \mathrm{Wh} / \mathrm{kg}$. Publication: ACS Energy Lett., 2019, 4 (5), 1073-1079

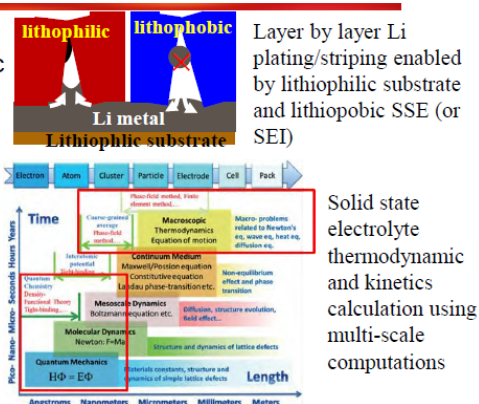

plating/striping enabled

by lithiophilic substrate and lithiopobic SSE (or

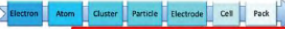

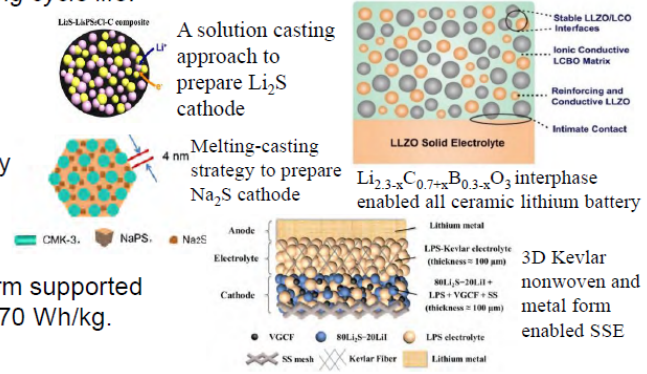

Chunsheng Wang

\section{Critical science gaps and opportunities}

Materials Science:

$>$ Promoting layer by layer Li plating/tripping: Lithiophilic substrate (Li-M alloy) and lithiophobic interphase/interface (LiF-rich) at Li/solid state electrolyte (SSE).

(1) $\mathrm{F}$ doped $\mathrm{Li}_{6} \mathrm{PS}_{5} \mathrm{Cl}_{0.3} \mathrm{~F}_{0.7}$ electrolyte with conductivity of $7.1 \times 10^{-4} \mathrm{~S} \mathrm{~cm}^{-1} @ \mathrm{RT}$ enabling a critical current of $6.4 \mathrm{~mA} \mathrm{~cm}^{-2}$ at a capacity of $5 \mathrm{mAh} \mathrm{cm}^{-2}$;

(2) Inserting a lithiophilic-lithiophobic gradient buffer layer between Li and SSE with lithiophilic side attaching to $\mathrm{Li}$ and lithionphobic side attaching to SSE. The buffer layer should be electrochemical stable with SSE.

$>$ Robust solid-solid interfaces : soft organic/polymer cathodes with strong ionic bonding to SSE enabling long cycle life; cathode potential: 2 V; Cathode energy density of $1674 \mathrm{Wh} \mathrm{kg}^{-1}$.
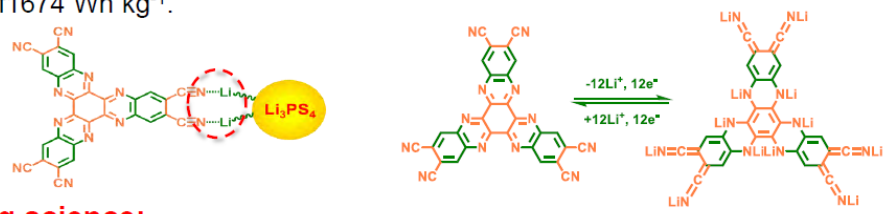

Processing science:

> A solution casting approach to prepare a thin electrolyte

> Melting-casting strategy to prepare a cathode

Battery Architecture:

> Robust solid-solid structure: 3D material supported cathode and electrolyte;

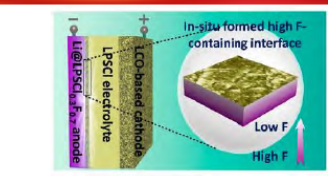

$\mathrm{Li}_{6} \mathrm{PS}_{5} \mathrm{Cl}_{0.3} \mathrm{~F}_{0.7} \mathrm{SSE}$ enabling formation of lithiophobic LiF after contacting Li (ACS Energy Lett. 2020, 5,1035-1043)

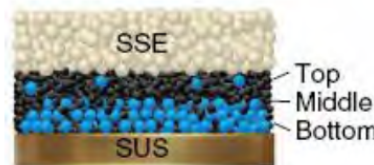

A lithiophilic-lithiophobic gradient buffer layer between Li and SSE (Nat. Energy, $5,2020,299)$
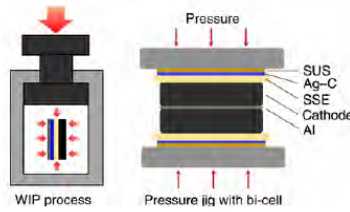

Presurzation using WIP Nat. Mater. 2019, 18, 1278-1291 


\section{Thomas Yersak (General Motors)}

\section{Introduction: General Motors R\&D}

- Scope of my programs/researchers

- Tom Yersak is a Senior Researcher in the Energy Storage Materials Group of the Chemical and Materials Systems Lab at GM R\&D. His group manager is Dr. Mei Cai and his lab director is Dr. Mark Verbrugge.

- Along with his peers, GM R\&D's solid-state research efforts span multiple classes of solid-state electrolyte.

- Tom's team of researchers focuses on the processing of solid-state batteries with sulfide glass electrolyte. His team's work is supported by DOE EERE BMR grant DE-

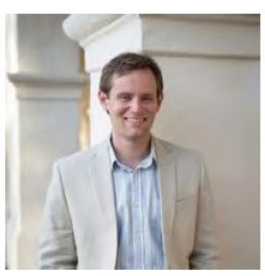
EE0008857.

What is unique to my capabilities, knowledge, resources

- Understanding of how sulfide glass composition affects functional properties such as rheology, moisture stability, ionic conductivity, and anodic/cathodic stabilities.

- Access to a pilot scale cell manufacturing line and a full suite of material characterization tools.

- What I recommend you read from my program

- Yersak, Thomas, et al. "Hot pressed, fiber-reinforced $\left(\mathrm{Li}_{2} \mathrm{~S}\right)_{70}\left(\mathrm{P}_{2} \mathrm{~S}_{5}\right)_{30}$ solid-state electrolyte separators for $\mathrm{Li}$ metal batteries." ACS Applied Energy Materials 2.5 (2019): 3523-3531.

- What I recommend you read from literature, the big aha's for me

- Han, Fudong, et al. "High electronic conductivity as the origin of lithium dendrite formation within solid electrolytes." Nature Energy 4.3 (2019): 187-196.

- Masias, Alvaro, et al. "Elastic, plastic, and creep mechanical properties of lithium metal." Journal of materials science 54.3 (2019): 2585-2600.

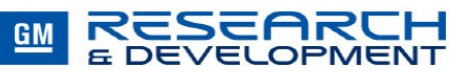

\section{Critical Science Gaps and Opportunities for SSBS}

Moisture stability

- For any SSB technology to be viable, it must be capable of being manufactured in a dry room.

- At ambient conditions sulfide SSEs hydrolyze and LLZO's surface passivates with $\mathrm{Li}_{2} \mathrm{CO}_{3}$ and $\mathrm{LiOH}$.

- How are the functional properties of SSEs affected by exposure to dry room conditions?

- What strategies can be deployed to mitigate the degradation of SSEs during processing?
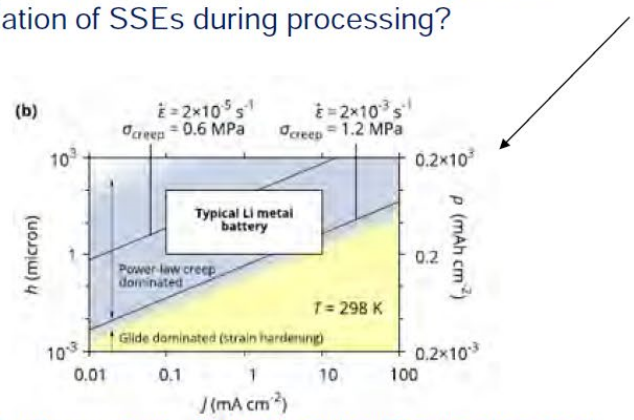

W.S. LePage et al. Journal of the Electrochemical Society, 166(2) A89 - A97 (2019).
Stack Pressure

- 2019 papers out of Oxford and U. of M. elucidate the need for a Li metal SSB stack pressure.

- Automotive OEM battery packs are designed to apply << $1 \mathrm{MPa}$ stack pressure to cells.

- SSBs afford system-level pack de-contenting (e.g. eliminate thermal mg'mt), however, the hardware required to apply a $1 \mathrm{MPa}$ stack pressure would add cost and mass.

- How can Li metal SSBs be designed to decrease the required stack pressure without impacting projected energy density?

- How does stack pressure affect the performance of non-zero strain cathode materials?

- How does stack pressure contribute to fatigue failure of cell components like thin SSE separators? 


\section{APPENDIX C. KEY TOPICS FROM SSB WORKSHOP}

\section{Cathodes and Cathode/SE Interfaces}

\section{What has been done}

- Structural characterization of NMC, LCO and other cathodes (e.g., volume expansion/contraction during cycling)

- Identification of challenges with solid-state cathodes

- Interface delamination from the electrolyte

- Interparticle fracture resulting in the loss of active material

- Total volume expansion

- Some challenges are similar to solid-oxide fuel cell cathodes (Waschman)

- There are engineering solutions from solid-oxide fuel cells that may be applicable to solid-state batteries

- There are significant challenges with the cation interdiffusion/crossover during high temperature sintering (Doeff)

- Demonstration of oxide coatings (e.g., $\mathrm{LiNbO}_{3}$ ) on cathode particles to improve electrochemical stability, especially with sulfide electrolytes. (Samsung)

Gaps in the science

- Defect equilibria and thermodynamic constants

- Tailoring of cathode and electrolyte compositions and structure to minimize stresses

- Zero-strain materials

- Soft cathodes (Chunsheng)

- Mismatch in mechanical properties of cathodes vs. SEs

- Interface modification to help accommodate volume expansion

- How to form robust interfaces without inducing cation interdiffusion/crossover?

- Better mechanical characterization of electrodes, electrolytes, and coupled systems

- Electro-chemo-mechanics, changes in electrochemical stimuli such as bias on bulk or micro/nano mechanical properties. How does hydrostatic pressure affect electrochemical potential of a cathode?

- How does charge transfer occur at solid-solid interfaces?

- What decomposition products are formed at electrode/SE interfaces? Are these reactions selflimiting? Answers will depend on material selection and possibly processing method.

\section{Li Metal Anodes}

What has been done

- Understanding of Li mechanics

- Plastic/elastic properties of the bulk

- Nanoscale properties via indentation and pillar compression

○ Strong when small

- Two different Li flow mechanisms, dependent on length scale

- Li penetration

- Direct observation of Li penetration in both polymers and ceramics

- Improved modeling of penetration by Barai, Srinivasen, Albertus

- $\quad$ Li stripping

- Stack pressure effective in mitigating rise in overpotential

- Li redistribution/confinement

- In planar geometry Li redistribution observed after thousands of cycles, results in eventual failure of thin film batteries 
- 3D geometries (Waschman, Doeff) can effectively help improve critical current densities and accommodate Li volume changes

Gaps in the science

- Understanding of homogenous Li plating and stripping

- The importance of wetting, 'lithiophobic' vs. "lithiophilic," which is more important for performance?

- Understanding of Li penetration and how to prevent it

- A closer look at Lipon and why it works is recommended

- Role of defects/impurities in Li

- Better correlation/validation between experiment and modeling

- Understanding stack pressure requirements

- Why does stack pressure help?

- How to reduce the stack pressure. Needs to be $<1 \mathrm{MPa}$ for practical cells

- $\quad$ Li confinement/redistribution

- What happens as large Li capacities are cycled thousands of times for a wide range of capacities, cell geometries and electrolytes

- 3D electrodes, what advantages do they offer?

- $\quad$ Li free cells

- Not well studied, but the optimal energy density.

- What is different/the same as a traditional cell

- Understanding of the mechanics of Li in confined spaces

- We know hardness effects are different at small length scales.

- How to study the confined Li?

- Does purity change mechanics?

\section{Solid Electrolyte Materials and Processing}

\section{What has been done}

- Development of new materials and synthesis routes for wide range of SEs

- Material classes: oxides, sulfides, polymers, composites, glasses, ionogels

- Synthesis routes: solid-state, solvent-mediated, mechanochemical, sputtering, aerosol methods (e.g., flame spray pyrolysis and RST)

- Demonstration of several solid electrolytes with very high conductivities comparable to liquid electrolytes (ca. 1-10 $\mathrm{mS} / \mathrm{cm}$ at room temperature)

- Development of composites with polymer binder (1-5\%) to fabricate thin SE layers $(<30 \mu \mathrm{m})$

- Drawback: significantly lower conductivity compared to pure ceramic/glass SEs

- Demonstration of processing routes to produce SE layers $<30 \mu \mathrm{m}$ thick

- Solution casting

- Melt casting

- Sputtering

- $\quad$ Spin coating

- Several studies demonstrating the efficacy of fiber reinforcement

Gaps in the science

- How to address air/moisture sensitivity of certain materials (e.g., sulfides, LLZO)?

- How to incorporate cathodes and polymer binders with SEs which require high temperature sintering?

- Some new processing routes (e.g., warm isostatic pressing) are batch and do not necessarily have rollto-roll analogue

- What is best way to incorporate SE in composite cathodes? 


\section{Cell Architecture: Key driver for industry—Less stuff = lower cost}

What has been done

- Not a lot

- Goal is to improve cell level energy density and reduce packaging and thermal management systems if viable.

- External pressure increases battery weight and cost. Pressures need to be $<1 \mathrm{MPa}$

- Samsung paper demonstrated several cell stacks

- Engineering starts at the cell level and moves to cell stacks and battery packs

Gaps in the science

- How to reduce required pressure

- Strain engineering within the cell may reduce need for external pressure

- Importance of binders

- Can we design binder-less processing?

- Can solid-state be done with dry processing?

- Can fiber reinforcements help enable better cell performance/different cell designs?

- Lots of $\mathrm{Li} / \mathrm{SE} / \mathrm{Li}$ cycling data in the literature, but integrating SEs with cathodes to produce full cells is much more difficult

- What is the best way to develop cell stacks?

- Sandwiching Li layers?

- What effect will this have in solid-state with Li expansion and contraction?

- What is the optimal cell geometry to minimize requirements of external pressure?

- Cylindrical vs. pouch cells. Something new?

- How will solid-state cells hold up to abuse fatigue over many years of operation?

- What is the optimal layout for solid-state battery (SSB) production lines?

- On one hand, want to use already in-place lithium-ion battery manufacturing lines

- On other hand, SSBs may enable unique ways of fabricating cathodes to obtain performance advantages (e.g., dry processing methods, making 'fat' cathodes) 
